\title{
Looking for New Naturally Aligned Higgs Doublets at the LHC
}

\section{P. S. Bhupal Dev}

Consortium for Fundamental Physics, School of Physics and Astronomy, University of Manchester, Manchester M13 9PL, United Kingdom.

Physik Department T30d, Technische Universität München, James-Franck-Straße 1, 85748

Garching, Germany.

E-mail: bhupal.devetum.de

\section{Apostolos Pilaftsis*}

Consortium for Fundamental Physics, School of Physics and Astronomy, University of Manchester, Manchester M13 9PL, United Kingdom.

CERN, Physics Department, Theory Division, CH-1211 Geneva 23, Switzerland.

E-mail: Apostolos.Pilaftsis@manchester.ac.uk

Since the current LHC Higgs data suggest the couplings of the observed $125 \mathrm{GeV}$ Higgs boson to be close to the Standard Model (SM) expectations, any extended Higgs sector must lead to the so-called SM alignment limit, where one of the Higgs bosons behaves exactly like that of the SM. In the context of the Two Higgs Doublet Model (2HDM), this alignment is often associated with either decoupling of the heavy Higgs sector or accidental cancellations in the 2HDM potential. We present a novel symmetry justification for 'natural' alignment without necessarily decoupling or fine-tuning. We show that there exist only three different symmetry realizations of the natural alignment scenario in 2HDM. We analyze new collider signals for the heavy Higgs sector in the natural alignment limit, which dominantly lead to third-generation quarks in the final state and can serve as a useful observational tool during the Run-II phase of the LHC.

18th International Conference From the Planck Scale to the Electroweak Scale 25-29 May 2015

Ioannina, Greece

${ }^{*}$ Speaker. 


\section{Introduction}

The discovery of a Higgs boson in the Run-I phase of the LHC [1] provides the first experimental evidence for the Higgs mechanism [2] as the standard theory of electroweak symmetry breaking (EWSB). The measured couplings of the discovered Higgs boson are remarkably consistent with those predicted by the Standard Model (SM) [3]. However, the possibility of an extended Higgs sector, as suggested by various well-motivated new-physics scenarios, such as supersymmetry, is still allowed by the current experimental data.

Here we consider the simplest extension of the standard Higgs mechanism, namely the Two Higgs Doublet Model (2HDM) [4], where the SM Higgs doublet is supplemented by another isodoublet with hypercharge $Y=1$. In the doublet field space $\Phi_{1,2}$, where $\Phi_{i}=\left(\phi_{i}^{+}, \phi_{i}^{0}\right)^{\top}$, the general 2HDM potential reads

$$
\begin{aligned}
V= & -\mu_{1}^{2}\left(\Phi_{1}^{\dagger} \Phi_{1}\right)-\mu_{2}^{2}\left(\Phi_{2}^{\dagger} \Phi_{2}\right)-\left[m_{12}^{2}\left(\Phi_{1}^{\dagger} \Phi_{2}\right)+\text { H.c. }\right] \\
& +\lambda_{1}\left(\Phi_{1}^{\dagger} \Phi_{1}\right)^{2}+\lambda_{2}\left(\Phi_{2}^{\dagger} \Phi_{2}\right)^{2}+\lambda_{3}\left(\Phi_{1}^{\dagger} \Phi_{1}\right)\left(\Phi_{2}^{\dagger} \Phi_{2}\right)+\lambda_{4}\left(\Phi_{1}^{\dagger} \Phi_{2}\right)\left(\Phi_{2}^{\dagger} \Phi_{1}\right) \\
& +\left[\frac{\lambda_{5}}{2}\left(\Phi_{1}^{\dagger} \Phi_{2}\right)^{2}+\lambda_{6}\left(\Phi_{1}^{\dagger} \Phi_{1}\right)\left(\Phi_{1}^{\dagger} \Phi_{2}\right)+\lambda_{7}\left(\Phi_{1}^{\dagger} \Phi_{2}\right)\left(\Phi_{2}^{\dagger} \Phi_{2}\right)+\text { H.c. }\right],
\end{aligned}
$$

which contains four real mass parameters $\mu_{1,2}^{2}, \operatorname{Re}\left(m_{12}^{2}\right), \operatorname{Im}\left(m_{12}^{2}\right)$, and ten real quartic couplings $\lambda_{1,2,3,4}, \operatorname{Re}\left(\lambda_{5,6,7}\right)$, and $\operatorname{Im}\left(\lambda_{5,6,7}\right)$. Thus, the vacuum structure of the general $2 \mathrm{HDM}$ can be quite rich [5], as compared to the SM.

The quark-sector Yukawa Lagrangian in the general 2HDM is given by

$$
-\mathscr{L}_{Y}^{q}=\bar{Q}_{L}\left(h_{1}^{d} \Phi_{1}+h_{2}^{d} \Phi_{2}\right) d_{R}+\bar{Q}_{L}\left(h_{1}^{u} \widetilde{\Phi}_{1}+h_{2}^{u} \widetilde{\Phi}_{2}\right) u_{R},
$$

where $\widetilde{\Phi}_{i}=\mathrm{i} \sigma^{2} \Phi_{i}^{*}\left(\sigma^{2}\right.$ being the second Pauli matrix) are the isospin conjugates of $\Phi_{i}, Q_{L}=$ $\left(u_{L}, d_{L}\right)^{\top}$ are the $S U(2)_{L}$ quark doublets and $u_{R}, d_{R}$ are right-handed quark singlets. To avoid potentially large flavor-changing neutral current processes at the tree level induced by the Yukawa interactions in (1.2), one imposes a discrete $Z_{2}$ symmetry [4] under which

$$
\Phi_{1} \rightarrow-\Phi_{1}, \quad \Phi_{2} \rightarrow \Phi_{2}, \quad u_{R a} \rightarrow u_{R a}, \quad d_{R a} \rightarrow d_{R a} \text { or } d_{R a} \rightarrow-d_{R a}
$$

( $a=1,2,3$ being the quark family index) so that only $\Phi_{2}$ gives mass to up-type quarks, and only $\Phi_{1}$ or only $\Phi_{2}$ gives mass to down-type quarks. The $Z_{2}$ symmetry (1.3) is satisfied by four discrete choices of tree-level Yukawa couplings between the Higgs doublets and SM fermions, which are known as the Type I, II, X (lepton-specific) and Y (flipped) 2HDMs [4]. Global fits to the LHC Higgs data (see e.g., $[6,7]$ ) suggest that all four types of $2 \mathrm{HDMs}$ with natural flavor conservation are constrained to lie close to the so-called SM alignment limit [8-12], where the mass eigenbasis of the CP-even scalar sector aligns with the SM gauge eigenbasis.

The SM alignment is often associated with the decoupling limit, in which all the non-standard Higgs bosons are assumed to be much heavier than the electroweak scale so that the lightest CPeven scalar behaves just like the SM Higgs boson. The alignment limit can also be achieved, without decoupling $[9,10,13]$, but for small $\tan \beta$ values, this is usually attributed to accidental cancellations in the $2 \mathrm{HDM}$ potential. We present a novel symmetry argument to naturally justify the alignment limit [11], independently of the kinematic parameters of the theory, such as the 
heavy Higgs mass and $\tan \beta$. In Section 3, we show that there exist only three possible symmetry realizations of the scalar potential which predict natural alignment, as defined in Section 2. We explicitly analyze the simplest case, namely, the Maximally Symmetric 2HDM (MS-2HDM), which realizes a SO(5) symmetry in the bilinear field basis to be discussed in Section 3. We show that the renormalization group (RG) effects due to the hypercharge gauge coupling $g^{\prime}$ and third-generation Yukawa couplings, as well as soft-breaking mass parameter $m_{12}^{2}$, induce relevant deviations from the $\mathrm{SO}(5)$ limit, which lead to distinct predictions for the Higgs spectrum of the MS-2HDM (see Section 4). In particular, a striking feature of the $\mathrm{SO}(5)$ limit is that the heavy Higgs sector is predicted to be quasi-degenerate, apart from being gaugephobic, which is a generic feature in the alignment limit. Moreover, the current experimental constraints force the heavy Higgs sector to lie above the top-quark threshold in the MS-2HDM. Thus, the dominant collider signal for this sector involves final states with third-generation quarks. We make a parton-level study of some of these signals (see Section 5), which can be useful for the heavy Higgs searches in the ongoing Run-II phase of the LHC.

\section{Natural Alignment Condition}

For simplicity, we consider the CP-conserving 2HDM, but our results can be easily generalized to the CP-violating case. After EWSB by the vacuum expectation values (VEVs) $v_{1,2}$ of the two scalar fields $\Phi_{1,2}$, there are five physical scalar mass eigenstates: two CP-even $(h, H)$, one CP-odd (a) and two charged $\left(h^{ \pm}\right)$scalars. The corresponding mass eigenvalues are given by [14]

$$
\begin{aligned}
& M_{h^{ \pm}}^{2}=\frac{m_{12}^{2}}{s_{\beta} c_{\beta}}-\frac{v^{2}}{2}\left(\lambda_{4}+\lambda_{5}\right)+\frac{v^{2}}{2 s_{\beta} c_{\beta}}\left(\lambda_{6} c_{\beta}^{2}+\lambda_{7} s_{\beta}^{2}\right), \quad M_{a}^{2}=M_{h^{ \pm}}^{2}+\frac{v^{2}}{2}\left(\lambda_{4}-\lambda_{5}\right), \\
& M_{H}^{2}=\frac{1}{2}\left[(A+B)-\sqrt{(A-B)^{2}+4 C^{2}}\right], \quad M_{h}^{2}=\frac{1}{2}\left[(A+B)+\sqrt{(A-B)^{2}+4 C^{2}}\right],
\end{aligned}
$$

where we have used the short-hand notations $c_{\beta} \equiv \cos \beta$ and $s_{\beta} \equiv \sin \beta$ with $\tan \beta=v_{2} / v_{1}$ and

$$
\begin{aligned}
& A=M_{a}^{2} s_{\beta}^{2}+v^{2}\left(2 \lambda_{1} c_{\beta}^{2}+\lambda_{5} s_{\beta}^{2}+2 \lambda_{6} s_{\beta} c_{\beta}\right), \\
& B=M_{a}^{2} c_{\beta}^{2}+v^{2}\left(2 \lambda_{2} s_{\beta}^{2}+\lambda_{5} c_{\beta}^{2}+2 \lambda_{7} s_{\beta} c_{\beta}\right), \\
& C=-M_{a}^{2} s_{\beta} c_{\beta}+v^{2}\left(\lambda_{34} s_{\beta} c_{\beta}+\lambda_{6} c_{\beta}^{2}+\lambda_{7} s_{\beta}^{2}\right) .
\end{aligned}
$$

with $\lambda_{34}=\lambda_{3}+\lambda_{4}$. The mixing between the mass eigenstates in the CP-odd and charged sectors is governed by the angle $\beta$, whereas in the CP-even sector, it is governed by the angle $\alpha=(1 / 2) \tan ^{-1}[2 C /(A-B)]$. The SM Higgs field can be identified as the linear combination

$$
H_{\mathrm{SM}}=H \cos (\beta-\alpha)+h \sin (\beta-\alpha) .
$$

Thus, the couplings of $h$ and $H$ to the SM gauge bosons $V=W^{ \pm}, Z$ with respect to the SM Higgs couplings $g_{H_{\mathrm{SM} V} V}$ will be respectively $\sin (\beta-\alpha)$ and $\cos (\beta-\alpha)$. The $S M$ alignment limit is defined as $\alpha \rightarrow \beta$ (or $\alpha \rightarrow \beta-\pi / 2$ ) when $H(h)$ couples to vector bosons exactly as in the SM, whereas $h(H)$ becomes gaugephobic. For concreteness, we will take the alignment limit as $\alpha \rightarrow \beta$. 
To derive the alignment condition, we rewrite the CP-even scalar mass matrix as

$$
M_{S}^{2}=\left(\begin{array}{ll}
A & C \\
C & B
\end{array}\right)=\left(\begin{array}{cc}
c_{\beta} & -s_{\beta} \\
s_{\beta} & c_{\beta}
\end{array}\right)\left(\begin{array}{cc}
\widehat{A} & \widehat{C} \\
\widehat{C} & \widehat{B}
\end{array}\right)\left(\begin{array}{cc}
c_{\beta} & s_{\beta} \\
-s_{\beta} & c_{\beta}
\end{array}\right),
$$

where $\widehat{A}=2 v^{2}\left[c_{\beta}^{4} \lambda_{1}+s_{\beta}^{2} c_{\beta}^{2} \lambda_{345}+s_{\beta}^{4} \lambda_{2}+2 s_{\beta} c_{\beta}\left(c_{\beta}^{2} \lambda_{6}+s_{\beta}^{2} \lambda_{7}\right)\right]$,

$$
\begin{aligned}
& \widehat{B}=M_{a}^{2}+\lambda_{5} v^{2}+2 v^{2}\left[s_{\beta}^{2} c_{\beta}^{2}\left(\lambda_{1}+\lambda_{2}-\lambda_{345}\right)-s_{\beta} c_{\beta}\left(c_{\beta}^{2}-s_{\beta}^{2}\right)\left(\lambda_{6}-\lambda_{7}\right)\right] \\
& \widehat{C}=v^{2}\left[s_{\beta}^{3} c_{\beta}\left(2 \lambda_{2}-\lambda_{345}\right)-c_{\beta}^{3} s_{\beta}\left(2 \lambda_{1}-\lambda_{345}\right)+c_{\beta}^{2}\left(1-4 s_{\beta}^{2}\right) \lambda_{6}+s_{\beta}^{2}\left(4 c_{\beta}^{2}-1\right) \lambda_{7}\right],
\end{aligned}
$$

and we have used the short-hand notation $\lambda_{345} \equiv \lambda_{3}+\lambda_{4}+\lambda_{5}$. Evidently, the SM alignment limit $\alpha \rightarrow \beta$ is obtained when $\widehat{C}=0$ in (2.4) [9]. This yields the quartic equation

$$
\lambda_{7} \tan ^{4} \beta-\left(2 \lambda_{2}-\lambda_{345}\right) \tan ^{3} \beta+3\left(\lambda_{6}-\lambda_{7}\right) \tan ^{2} \beta+\left(2 \lambda_{1}-\lambda_{345}\right) \tan \beta-\lambda_{6}=0 .
$$

For natural alignment, (2.6) should be satisfied for any value of $\tan \beta$, which requires the coefficients of the polynomial in $\tan \beta$ to vanish identically [11]. This implies

$$
2 \lambda_{1}=2 \lambda_{2}=\lambda_{345}, \quad \lambda_{6}=\lambda_{7}=0 .
$$

In particular, for $\lambda_{6}=\lambda_{7}=0$ as in the $Z_{2}$-symmetric 2HDMs, (2.6) has a solution

$$
\tan ^{2} \beta=\frac{2 \lambda_{1}-\lambda_{345}}{2 \lambda_{2}-\lambda_{345}}>0,
$$

independent of $M_{a}$. After some algebra, the simple solution (2.8) to our natural alignment condition (2.6) can be shown to be equivalent to that derived in [10].

\section{Symmetry Classifications of the 2HDM Potential}

To identify all accidental symmetries of the 2HDM potential (1.1), it is convenient to work in the bilinear scalar field formalism [15] by introducing an 8-dimensional complex multiplet $\boldsymbol{\Phi} \equiv$ $\left(\Phi_{1}, \Phi_{2}, \widetilde{\Phi}_{1}, \widetilde{\Phi}_{2}\right)^{\top}[5,16,17]$. In terms of the $\boldsymbol{\Phi}$-multiplet, we define a null 6-dimensional Lorentz vector $R^{A} \equiv \boldsymbol{\Phi}^{\dagger} \Sigma^{A} \boldsymbol{\Phi}$, where $A=0,1, \ldots, 5$ and the six $8 \times 8$-dimensional matrices $\Sigma^{A}$ may be expressed in terms of the three Pauli matrices $\sigma^{1,2,3}$ and the identity matrix $\mathbf{1}_{2 \times 2} \equiv \sigma^{0}$, as follows:

$$
\begin{aligned}
& \Sigma^{0,1,3}=\frac{1}{2} \sigma^{0} \otimes \sigma^{0,1,3} \otimes \sigma^{0}, \quad \Sigma^{2}=\frac{1}{2} \sigma^{3} \otimes \sigma^{2} \otimes \sigma^{0}, \\
& \Sigma^{4}=-\frac{1}{2} \sigma^{2} \otimes \sigma^{2} \otimes \sigma^{0}, \quad \Sigma^{5}=-\frac{1}{2} \sigma^{1} \otimes \sigma^{2} \otimes \sigma^{0} .
\end{aligned}
$$

Note that the bilinear field space spanned by the 6-vector $R^{A}$ realizes an orthochronous $\mathrm{SO}(1,5)$ symmetry group $[5,17]$.

In terms of the null-vector $R^{A}$, the $2 \mathrm{HDM}$ potential (1.1) takes on a simple quadratic form:

$$
V=-\frac{1}{2} M_{A} R^{A}+\frac{1}{4} L_{A B} R^{A} R^{B}
$$

where $M_{A}$ and $L_{A B}$ are $\mathrm{SO}(1,5)$ constant 'tensors' that depend on the mass parameters and quartic couplings given in (1.1) and their explicit forms may be found in $[17,18]$. Requiring that the $\mathrm{SU}(2)_{L}$ 


\begin{tabular}{c|ccccccccc}
\hline symmetry & $\mu_{1}^{2}$ & $\mu_{2}^{2}$ & $m_{12}^{2}$ & $\lambda_{1}$ & $\lambda_{2}$ & $\lambda_{3}$ & $\lambda_{4}$ & $\operatorname{Re}\left(\lambda_{5}\right)$ & $\lambda_{6}=\lambda_{7}$ \\
\hline$Z_{2} \times \mathrm{O}(2)$ & - & - & Real & - & - & - & - & - & Real \\
$\left(Z_{2}\right)^{2} \times \mathrm{SO}(2)$ & - & - & 0 & - & - & - & - & - & 0 \\
$\left(Z_{2}\right)^{3} \times \mathrm{O}(2)$ & - & $\mu_{1}^{2}$ & 0 & - & $\lambda_{1}$ & - & - & - & 0 \\
$\mathrm{O}(2) \times \mathrm{O}(2)$ & - & - & 0 & - & - & - & - & 0 & 0 \\
$Z_{2} \times[\mathrm{O}(2)]^{2}$ & - & $\mu_{1}^{2}$ & 0 & - & $\lambda_{1}$ & - & - & $2 \lambda_{1}-\lambda_{34}$ & 0 \\
$\mathrm{O}(3) \times \mathrm{O}(2)$ & - & $\mu_{1}^{2}$ & 0 & - & $\lambda_{1}$ & - & $2 \lambda_{1}-\lambda_{3}$ & 0 & 0 \\
$\mathrm{SO}(3)$ & - & - & $\mathrm{Real}$ & - & - & - & - & $\lambda_{4}$ & Real \\
$Z_{2} \times \mathrm{O}(3)$ & - & $\mu_{1}^{2}$ & $\mathrm{Real}$ & - & $\lambda_{1}$ & - & - & $\lambda_{4}$ & Real \\
$\left(Z_{2}\right)^{2} \times \mathrm{SO}(3)$ & - & $\mu_{1}^{2}$ & 0 & - & $\lambda_{1}$ & - & - & $\pm \lambda_{4}$ & 0 \\
$\mathrm{O}(2) \times \mathrm{O}(3)$ & - & $\mu_{1}^{2}$ & 0 & - & $\lambda_{1}$ & $2 \lambda_{1}$ & - & 0 & 0 \\
$\mathrm{SO}(4)$ & - & - & 0 & - & - & - & 0 & 0 & 0 \\
$Z_{2} \times \mathrm{O}(4)$ & - & $\mu_{1}^{2}$ & 0 & - & $\lambda_{1}$ & - & 0 & 0 & 0 \\
$\mathrm{SO}(5)$ & - & $\mu_{1}^{2}$ & 0 & - & $\lambda_{1}$ & $2 \lambda_{1}$ & 0 & 0 & 0 \\
\hline
\end{tabular}

Table 1: Relations between the parameters of the $U(1)_{Y}$-invariant $2 \mathrm{HDM}$ potential (1.1) for the 13 accidental symmetries [17] in a diagonally reduced basis, where $\operatorname{Im}\left(\lambda_{5}\right)=0$ and $\lambda_{6}=\lambda_{7}$.

gauge-kinetic term of the $\Phi$-multiplet remains canonical restricts the allowed set of rotations from $\mathrm{SO}(1,5)$ to $\mathrm{SO}(5)$, where only the spatial components $R^{I}$ (with $I=1, \ldots, 5$ ) transform and the zeroth component $R^{0}$ remains invariant. Consequently, in the absence of the hypercharge gauge coupling and fermion Yukawa couplings, the maximal symmetry group of the $2 \mathrm{HDM}$ is $G_{2 \mathrm{HDM}}^{R}=\mathrm{SO}(5)$. Including all proper, improper and semi-simple subgroups of $\mathrm{SO}(5)$, the accidental symmetries for the 2HDM potential were completely classified in $[5,17]$, as shown in Table 1 . Here we have used a diagonally reduced basis [19], where $\operatorname{Im}\left(\lambda_{5}\right)=0$ and $\lambda_{6}=\lambda_{7}$, thus reducing the number of independent quartic couplings to seven. Each of the symmetries listed in Table 1 leads to certain constraints on the mass and/or coupling parameters. From Table 1, we find that there are only three symmetries, namely $\mathrm{Z}_{2} \times[\mathrm{O}(2)]^{2}, \mathrm{O}(3) \times \mathrm{O}(2)$, and $\mathrm{SO}(5)$, which satisfy the natural alignment condition given by (2.7). ${ }^{1}$ In what follows, we focus on the simplest realization of the SM alignment, namely, the MS-2HDM based on the SO(5) group [11].

\section{Maximally Symmetric 2HDM}

From Table 1, we see that the maximal symmetry group in the bilinear field space is $\mathrm{SO}(5)$, in which case the parameters of the 2HDM potential (1.1) satisfy the following relations:

$$
\begin{aligned}
& \mu_{1}^{2}=\mu_{2}^{2}, \quad m_{12}^{2}=0, \\
& \lambda_{2}=\lambda_{1}, \quad \lambda_{3}=2 \lambda_{1}, \quad \lambda_{4}=\operatorname{Re}\left(\lambda_{5}\right)=\lambda_{6}=\lambda_{7}=0,
\end{aligned}
$$

\footnotetext{
${ }^{1}$ In Type-I $2 \mathrm{HDM}$, there exists an additional possibility of realizing an exact $\mathrm{Z}_{2}$ symmetry [20] which leads to an exact alignment, i.e. in the context of the so-called inert 2HDM [21].
} 
Thus, the $2 \mathrm{HDM}$ potential (1.1) is parametrized by just a single mass parameter $\mu_{1}^{2}=\mu_{2}^{2} \equiv \mu^{2}$ and a single quartic coupling $\lambda_{1}=\lambda_{2}=\lambda_{3} / 2 \equiv \lambda$, as in the SM:

$$
V=-\mu^{2}\left(\left|\Phi_{1}\right|^{2}+\left|\Phi_{2}\right|^{2}\right)+\lambda\left(\left|\Phi_{1}\right|^{2}+\left|\Phi_{2}\right|^{2}\right)^{2}=-\frac{\mu^{2}}{2} \boldsymbol{\Phi}^{\dagger} \boldsymbol{\Phi}+\frac{\lambda}{4}\left(\boldsymbol{\Phi}^{\dagger} \boldsymbol{\Phi}\right)^{2} .
$$

Given the isomorphism of the Lie algebras $\mathrm{SO}(5) \sim \mathrm{Sp}(4)$, the maximal symmetry group of the $2 \mathrm{HDM}$ in the original $\boldsymbol{\Phi}$-field space is $\mathrm{G}_{2 \mathrm{HDM}}^{\boldsymbol{\Phi}}=\left[\mathrm{Sp}(4) / Z_{2}\right] \times \mathrm{SU}(2)_{L}[11,17]$ in the custodial symmetry limit of vanishing $g^{\prime}$ and fermion Yukawa couplings.

\subsection{Scalar Spectrum}

Using the parameter relations given by (4.1), we find from (2.1a) and (2.1b) that in the MS2HDM, the CP-even scalar $H$ has mass $M_{H}^{2}=2 \lambda_{2} v^{2}$, whilst the remaining four scalar fields, denoted hereafter as $h, a$ and $h^{ \pm}$, are massless. This is a consequence of the Goldstone theorem, since after electroweak symmetry breaking, $\mathrm{SO}(5) \stackrel{\left\langle\Phi_{1,2}\right\rangle \neq 0}{\longrightarrow} \mathrm{SO}(4)$. Thus, we identify $H$ as the SM-like Higgs boson with the mixing angle $\alpha=\beta$ [cf. (2.3)], i.e. the SM alignment limit can be naturally attributed to the $\mathrm{SO}(5)$ symmetry of the theory.

In the exact $\mathrm{SO}(5)$-symmetric limit, the scalar spectrum of the MS-2HDM is experimentally unacceptable. This is because the four massless pseudo-Goldstone particles, viz. $h, a$ and $h^{ \pm}$, have sizable couplings to the SM $Z$ and $W^{ \pm}$bosons, and could induce additional decay channels, such as $Z \rightarrow h a$ and $W^{ \pm} \rightarrow h^{ \pm} h$, which are experimentally excluded [22]. However, as we will see below, the $\mathrm{SO}(5)$ symmetry may be violated predominantly by RG effects due to $g^{\prime}$ and third-generation Yukawa couplings, as well as by soft $\mathrm{SO}(5)$-breaking mass parameters, thereby lifting the masses of these pseudo-Goldstone particles to be consistent with the experimental constraints.

\subsection{RG and Soft Breaking Effects}

To calculate the RG and soft-breaking effects in a technically natural manner, we assume that the $\mathrm{SO}(5)$ symmetry is realized at some high scale $\mu_{X}$ much above the electroweak scale. The physical mass spectrum at the electroweak scale is then obtained by the RG evolution of the 2HDM parameters given by (1.1). Using the two-loop RG equations (RGEs) given in Appendix A, we first examine the deviation of the Higgs spectrum from the $\mathrm{SO}(5)$-symmetric limit due to $g^{\prime}$ and Yukawa coupling effects, in the absence of the soft-breaking term. This is illustrated in Figure 1 for a typical choice of parameters in the Type-II realization of the 2HDM. We find that the RG-induced $g^{\prime}$ effects only lift the charged Higgs mass $M_{h^{ \pm}}$, while the corresponding Yukawa coupling effects also lift slightly the mass of the non-SM CP-even pseudo-Goldstone boson $h$. However, they still leave the CP-odd scalar $a$ massless, which can be identified as a $\mathrm{U}(1)_{\mathrm{PQ}}$ axion [23].

Therefore, $g^{\prime}$ and Yukawa coupling effects are not sufficient to yield a viable Higgs spectrum at the weak scale, starting from a $\mathrm{SO}(5)$-invariant boundary condition (4.1) at some high scale $\mu_{X}$. To minimally circumvent this problem, we include soft $\mathrm{SO}(5)$-breaking effects, by assuming a nonzero soft-breaking term $\operatorname{Re}\left(m_{12}^{2}\right)$. In the $\mathrm{SO}(5)$-symmetric limit for the scalar quartic couplings, but with $\operatorname{Re}\left(m_{12}^{2}\right) \neq 0$, we obtain the following mass spectrum [cf. (2.1a) and (2.1b)]:

$$
M_{H}^{2}=2 \lambda_{2} v^{2}, \quad M_{h}^{2}=M_{a}^{2}=M_{h^{ \pm}}^{2}=\frac{\operatorname{Re}\left(m_{12}^{2}\right)}{s_{\beta} c_{\beta}},
$$




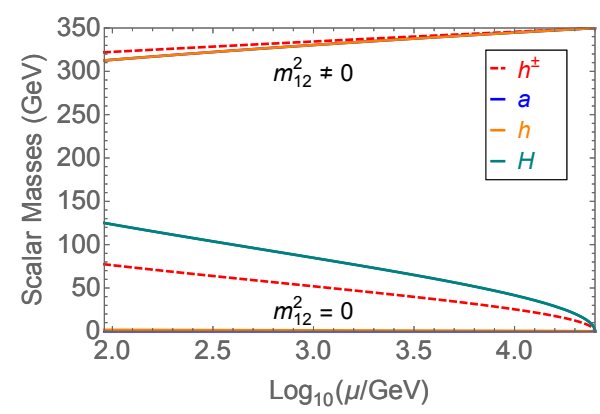

Figure 1: The scalar spectrum in the MS-2HDM without and with soft-breaking effects.

as well as an equality between the $\mathrm{CP}$-even and CP-odd mixing angles: $\alpha=\beta$, thus predicting an exact alignment for the SM-like Higgs boson $H$, simultaneously with an experimentally allowed heavy Higgs spectra (cf. Figure 1 for $m_{12}^{2} \neq 0$ case). Note that in the alignment limit, the heavy Higgs sector is exactly degenerate [cf. (4.3)] at the $\mathrm{SO}(5)$ symmetry-breaking scale, and at the low-energy scale, this degeneracy is mildly broken by the RG effects. Thus, we obtain a quasidegenerate heavy Higgs spectrum, which is a unique prediction of the MS-2HDM, valid even in the non-decoupling limit, and can be used to distinguish this model from other 2HDM scenarios.

\subsection{Misalignment Predictions}

As discussed in Section 4.2, there will be some deviation from the alignment limit in the lowenergy Higgs spectrum of the MS-2HDM due to RG and soft-breaking effects. By requiring that the mass and couplings of the SM-like Higgs boson $H$ are consistent with the LHC Higgs data [3], we derive predictions for the remaining scalar spectrum and compare them with the existing (in)direct limits on the heavy Higgs sector. We use the constraints in the $(\tan \beta, \beta-\alpha)$ plane derived from a recent global fit for the Type-II 2HDM [24], and require that for a given set of $\mathrm{SO}(5)$ boundary conditions $\left\{\mu_{X}, \tan \beta\left(\mu_{X}\right), \lambda\left(\mu_{X}\right)\right\}$, the RG-evolved 2HDM parameters at the weak scale must satisfy these alignment constraints on the lightest CP-even Higgs boson sector. This puts stringent constraints on the MS-2HDM parameter space, as shown in Figure 2 by the blue shaded region. In the red shaded region, there is no viable solution to the RGEs. We ensure that the remaining allowed (white) region satisfies the necessary theoretical constraints, i.e. positivity and vacuum stability of the Higgs potential, and perturbativity of the Higgs self-couplings [4]. From Figure 2, we find that there exists an upper limit of $\mu_{X} \lesssim 10^{9} \mathrm{GeV}$ on the SO(5)-breaking scale of the 2HDM potential, beyond which an ultraviolet completion of the theory must be invoked. The situation can be alleviated with the other two natural alignment scenarios listed in Table 1 and this will be the subject of a future publication.

For the allowed parameter space of our MS-2HDM as shown in Figure 2, we obtain concrete predictions for the remaining Higgs spectrum. In particular, the alignment condition imposes a lower bound on the soft breaking parameter $\operatorname{Re}\left(m_{12}^{2}\right)$, and hence, on the heavy Higgs spectrum. A comparison of the global fit limit on the charged Higgs-boson mass as a function of $\tan \beta$ [24] with our predicted limits from the alignment condition in the MS-2HDM for a typical value of the boundary scale $\mu_{X}=3 \times 10^{4} \mathrm{GeV}$ is shown in Figure 3 (left panel). It is clear that the alignment limits are stronger than the global fit limits, except in the very small and very large $\tan \beta$ regimes. 


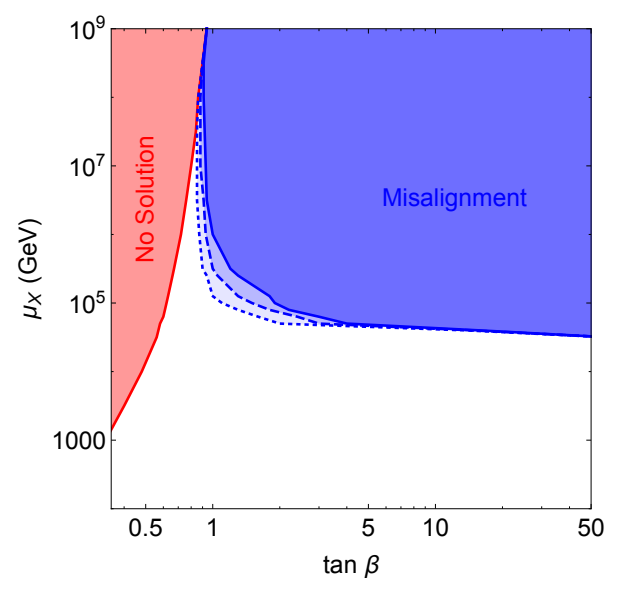

Figure 2: The $1 \sigma$ (dotted), $2 \sigma$ (dashed) and $3 \sigma$ (solid) exclusion contours (blue shaded region) from the alignment constraints in MS-2HDM. The red shaded region is theoretically excluded.
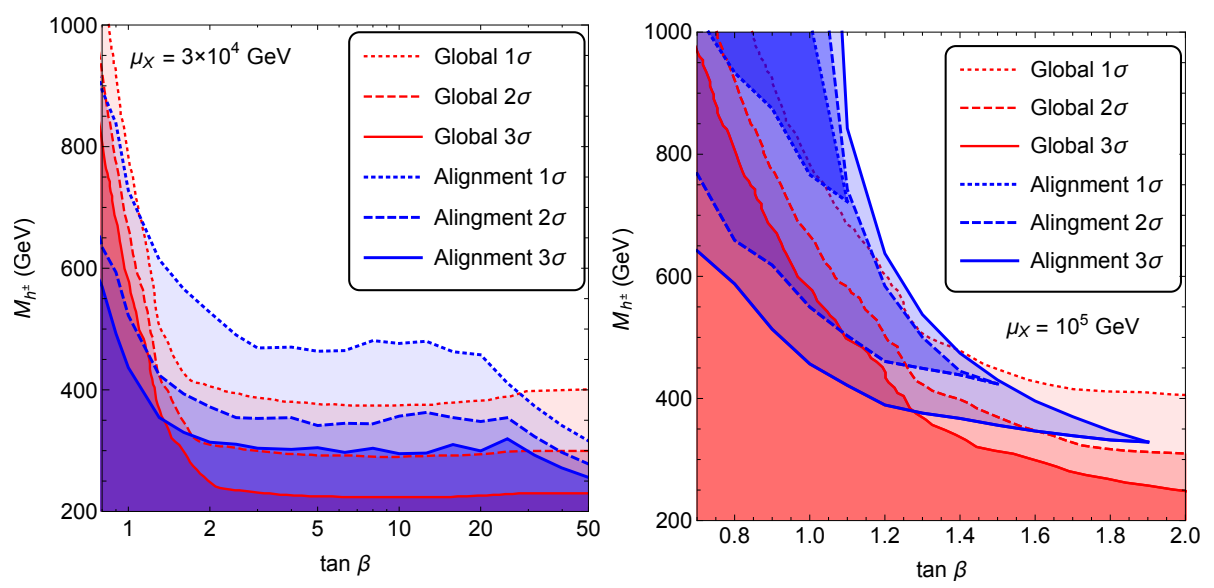

Figure 3: Left: The $1 \sigma$ (dotted), $2 \sigma$ (dashed) and $3 \sigma$ (solid) lower limits on the charged Higgs mass obtained from the alignment condition (blue lines) in the MS-2HDM with $\mu_{X}=3 \times 10^{4} \mathrm{GeV}$. Right: The $1 \sigma$ (dotted), $2 \sigma$ (dashed) and $3 \sigma$ (solid) allowed regions from the alignment condition (blue lines) for $\mu_{X}=10^{5} \mathrm{GeV}$. For comparison, the corresponding lower limits from a global fit are also shown (red lines).

From Figure 2, we note that for $\mu_{X} \gtrsim 10^{5} \mathrm{GeV}$, phenomenologically acceptable alignment is not possible in the MS-2HDM for large $\tan \beta$ and large $m_{12}^{2}$. Therefore, we also get an upper bound on the charged Higgs-boson mass $M_{h^{ \pm}}$from the misalignment condition, depending on $\tan \beta$. This is illustrated in Figure 3 (right panel) for $\mu_{X}=10^{5} \mathrm{GeV}$.

Similar alignment constraints are obtained for the heavy neutral pseudo-Goldstone bosons $h$ and $a$, which are predicted to be quasi-degenerate with the charged Higgs boson $h^{ \pm}$in the MS2HDM [cf. (4.3)]. The current experimental limits on the heavy neutral Higgs sector [22] are weaker than the alignment constraints in this case. Thus, the MS-2HDM scenario provides a natural reason for the absence of a heavy Higgs signal below the top-quark threshold, and this has important consequences for the heavy Higgs searches in the Run-II phase of the LHC, as discussed in Sec. 5. 

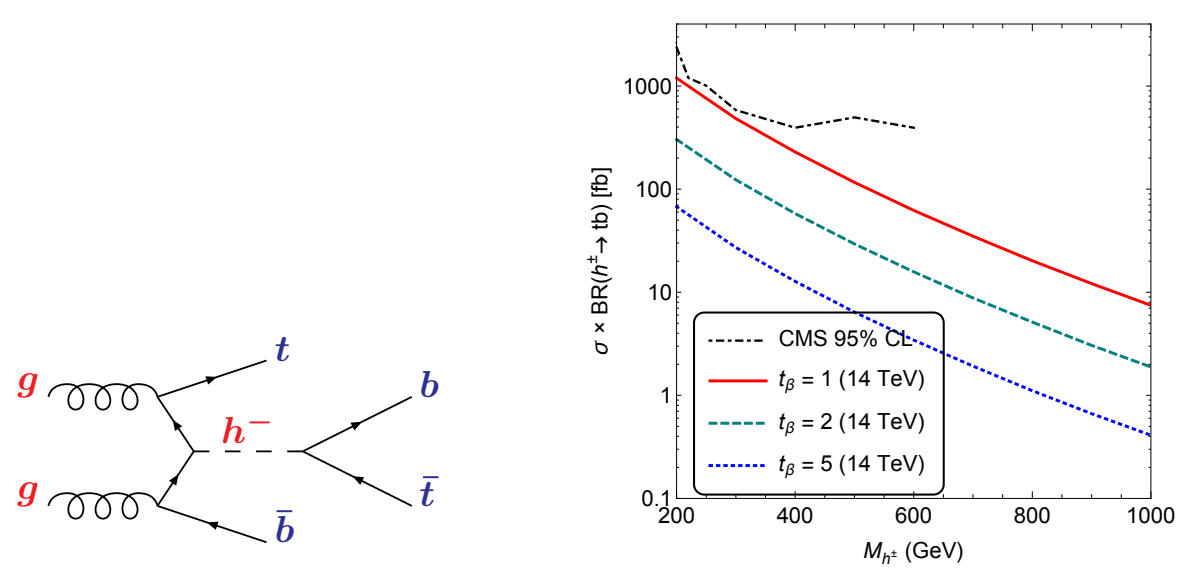

Figure 4: (Left) Feynman diagram and (right) production cross sections for the charged Higgs boson in the Type-II MS-2HDM at $\sqrt{s}=14 \mathrm{TeV}$ LHC. Also shown is the 95\% CL upper limit from the $\sqrt{s}=8 \mathrm{TeV}$ LHC data [25].

\section{Collider Signatures in the Alignment Limit}

In the alignment limit, the couplings of the lightest CP-even Higgs boson are exactly similar to the SM Higgs couplings, while the heavy CP-even Higgs boson is gaugephobic. Therefore, two of the relevant Higgs production mechanisms at the LHC, namely, the vector boson fusion and Higgsstrahlung processes are suppressed for the heavy neutral Higgs sector. As a consequence, the only relevant production channels to probe the neutral Higgs sector of the MS-2HDM are the gluon-gluon fusion and $t \bar{t} h(b \bar{b} h)$ associated production mechanisms at low (high) $\tan \beta$. For the charged Higgs sector of the MS-2HDM, the dominant production mode is the associated production process: $g g \rightarrow \bar{t} b h^{+}+t \bar{b} h^{-}$, irrespective of $\tan \beta$. Similarly, for the decay modes of the heavy neutral Higgs bosons in the MS-2HDM, the $t \bar{t}(b \bar{b})$ channel is the dominant one for low (high) $\tan \beta$ values, whereas for the charged Higgs boson $h^{+(-)}$, the $t \bar{b}(\bar{t} b)$ mode is the dominant one for any $\tan \beta$. Thus, the heavy Higgs sector of the MS-2HDM can be effectively probed at the LHC through the final states involving third-generation quarks.

\subsection{Charged Higgs Signal}

The most promising charged Higgs channel in the MS-2HDM is the $t \bar{t} b \bar{b}$ final state at the LHC, as illustrated in Figure 4 (left). Experimentally, this is a challenging mode due to large QCD backgrounds and the non-trivial event topology, involving at least four $b$-jets. Nevertheless, a recent CMS study [25] has presented a realistic analysis of this process with $\sqrt{s}=8 \mathrm{TeV}$ LHC data in the leptonic decay mode of the $W$ 's coming from top decay: $g g \rightarrow h^{ \pm} t b \rightarrow\left(\ell v_{\ell} b b\right)\left(\ell^{\prime} v_{\ell^{\prime}} b\right) b$ (with $\ell, \ell^{\prime}=e, \mu$ ). The resulting 95\% CL upper limit on the production cross section of $g g \rightarrow h^{ \pm} t b$ times the branching ratio of $h^{ \pm} \rightarrow t b$ is shown in Figure 4 (right) as a function of $M_{h^{ \pm}}$. We also show the corresponding predictions at $\sqrt{s}=14 \mathrm{TeV}$ LHC in the Type-II MS-2HDM for some representative values of $\tan \beta$. The cross section predictions were obtained at leading order (LO) by implementing the 2HDM Lagrangian in MadGraph5 [26] and using the NNPDF 2.3 parton distribution functions (PDFs) [27]. Our results in Figure 4 (right) suggest that the Run-II phase of the LHC could probe the low $\tan \beta$ region of the MS-2HDM parameter space using this process. 

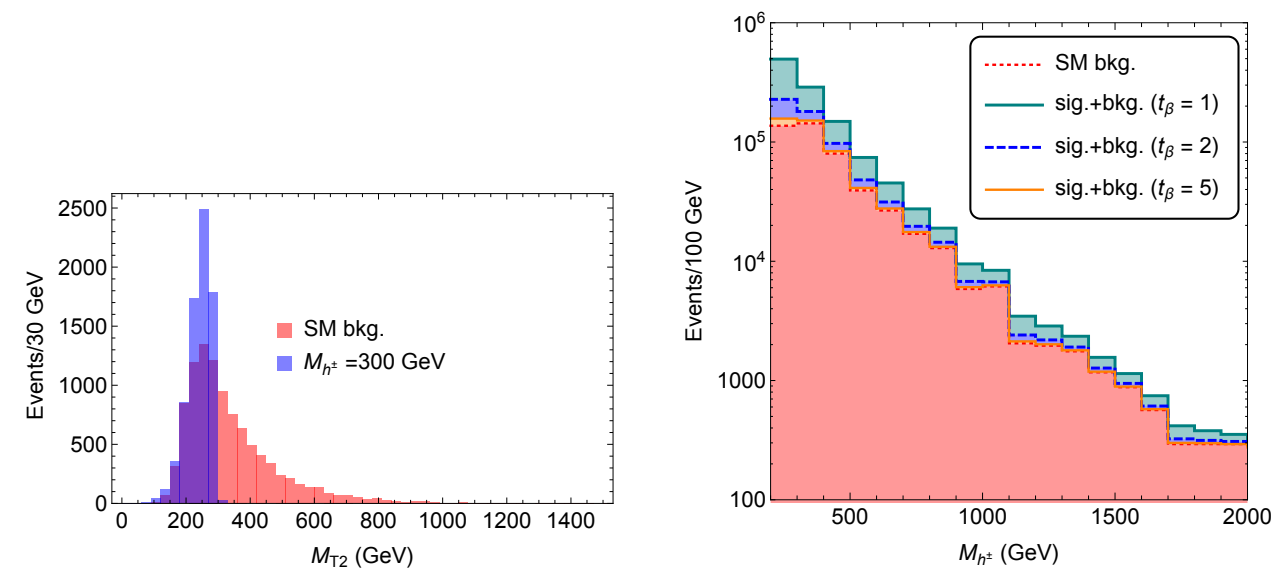

Figure 5: (Left) Charged Higgs boson mass reconstruction using the $M_{T 2}$ variable. (Right) The $t \bar{t} b \bar{b}$ signal in the MS-2HDM at $\sqrt{s}=14 \mathrm{TeV}$ LHC with $300 \mathrm{fb}^{-1}$ integrated luminosity.

In order to make a rough estimate of the $\sqrt{s}=14 \mathrm{TeV}$ LHC sensitivity to the charged Higgs signal, we also perform a parton-level simulation of the $t \bar{t} b \bar{b}$ signal and background events [11] (see also [28]). The inclusive SM cross section for $p p \rightarrow t \bar{t} b \bar{b}+X$ is $\sim 18 \mathrm{pb}$ at next-to-LO (NLO), with roughly $30 \%$ uncertainty due to higher order QCD corrections [29]. Most of the QCD background for the $t \bar{t} b \bar{b}$ final state can be reduced significantly by reconstructing at least one top-quark. The remaining irreducible background due to $\mathrm{SM} t \bar{t} b \bar{b}$ production can be suppressed with respect to the signal by reconstructing the charged Higgs boson mass, once a valid signal region is defined, e.g. in terms of an observed excess of events at the LHC in future. For the semi-leptonic decay mode of top-quarks leading to $4 b+2 \ell+\mathbb{E}_{T}$ final state, one cannot directly use an invariant mass observable to infer $M_{h^{ \pm}}$. A more useful quantity in this case is [30]

$$
M_{T 2}=\min _{\left\{\mathbf{p}_{T_{\mathrm{a}}}+\mathbf{p}_{T_{\mathrm{b}}}=\mathbf{p}_{T}\right\}}\left[\max \left\{m_{T_{\mathrm{a}}}, m_{T_{\mathrm{b}}}\right\}\right],
$$

where $\{a\},\{b\}$ stand for the two sets of particles in the final state, each containing a neutrino with part of the missing transverse momentum $\left(\mathbf{p}_{T_{\mathrm{a}, \mathrm{b}}}\right)$. Minimization over all possible sums of these two momenta gives the observed missing transverse momentum $\boldsymbol{p}_{T}$, whose magnitude is the same as $\mathbb{E}_{T}$ in our specific case. In (5.1), $m_{T_{i}}$ (with $i=\mathrm{a}, \mathrm{b}$ ) is the usual transverse mass variable for the system $\{i\}$. For the correct combination of the final state particles, viz. $\{a\}=\left(\ell v_{\ell} b b\right)$ and $\{\mathrm{b}\}=\left(\ell^{\prime} v_{\ell^{\prime}} b b\right)$ in (5.1), the maximum value of $M_{T 2}$ represents the charged Higgs boson mass, with the $M_{T 2}$ distribution smoothly dropping to zero at this point. This is illustrated in Figure 5 (left) for a typical choice of $M_{h^{ \pm}}=300 \mathrm{GeV}$. For comparison, we also show the $M_{T 2}$ distribution for the SM background, which obviously does not have a sharp endpoint. Thus, for a given hypothesized signal region defined in terms of an excess due to $M_{h^{ \pm}}$, we may impose an additional cut on $M_{T 2} \leq M_{h^{ \pm}}$ to enhance the signal-to-background ratio.

Assuming that the charged Higgs boson mass can be reconstructed efficiently, we present an estimate of the signal and background for the charged Higgs signal in MS-2HDM at $\sqrt{s}=14 \mathrm{TeV}$ LHC with $300 \mathrm{fb}^{-1}$ for some typical values of $\tan \beta$ in Figure 5 (right). We find that $M_{h^{ \pm}}$values up to about $2 \mathrm{TeV}$ can be probed via the $t \bar{t} b \bar{b}$ final state for low values of $\tan \beta$. 

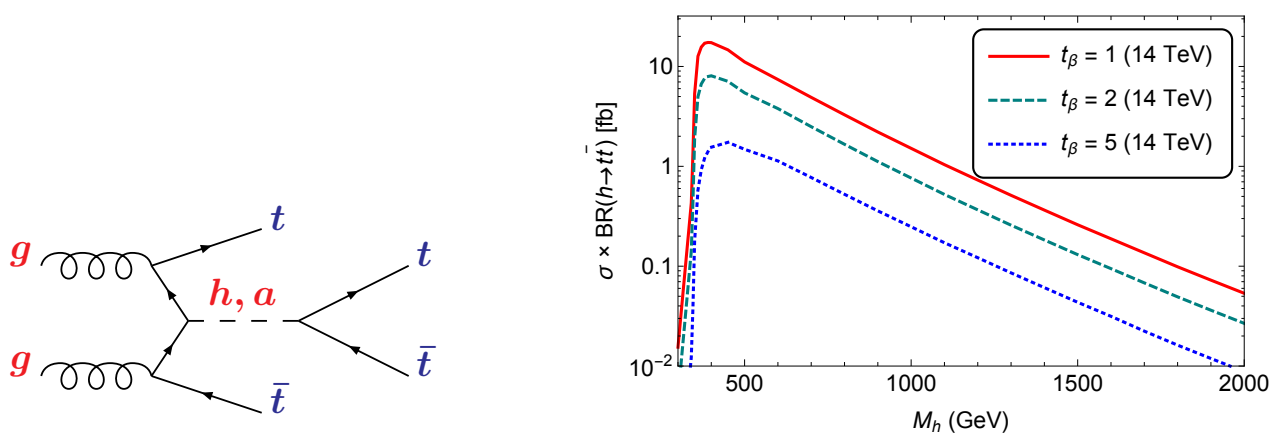

Figure 6: (Left) Feynman diagram and (right) four-top production cross section in the Type-II MS-2HDM at $\sqrt{s}=14 \mathrm{TeV}$ LHC for various values of $\tan \beta$.

\subsection{Heavy Neutral Higgs Signal}

So far there have been no direct searches for heavy neutral Higgs bosons involving $t \bar{t}$ and/or $b \bar{b}$ final states, mainly due to the challenges associated with uncertainties in the jet energy scales and the combinatorics arising from complicated multiparticle final states in a busy QCD environment. Nevertheless, these channels become pronounced in the MS-2HDM scenario, and hence, we have made a first attempt to study them in [11]. In particular, we focus on the search channel $g g \rightarrow$ $t \bar{t} h \rightarrow t \bar{t} t \bar{t}$, as shown in Figure 6 (left). A more sophisticated analysis of this four-top signal, e.g. including hadron-level simulation with jet clustering, flavo and top tagging with jet substructure, detector acceptance and momentum resolution effects at the LHC can be found in [31].

To get a rough estimate of the signal to background ratio for our four-top signal in the MS2HDM, we perform a parton-level simulation of the signal and background events at LO in QCD using MadGraph5 [26] with NNPDF 2 . 3 PDF sets [27]. For the inclusive SM cross section for the four-top final state at $\sqrt{s}=14 \mathrm{TeV}$ LHC, we obtain $11.85 \mathrm{fb}$, whereas our proposed signal cross sections are found to be comparable or smaller depending on $M_{h}$ and $\tan \beta$, as shown in Figure 6 (right). However, since we expect one of the $t \bar{t}$ pairs coming from an on-shell $h$ decay to have an invariant mass around $M_{h}$, we can use this information to significantly boost the signal over the irreducible SM background. Note that all the predicted cross sections shown in Figure 6 (right) are consistent with the current experimental upper bound [32].

Depending on the $W$ decay mode from $t \rightarrow W b$, there are 35 final states for four top decays. Experimentally, the most favored channel is the semi-leptonic/hadronic final state with two samesign isolated leptons: $g g \rightarrow t \bar{t} h \rightarrow(t \bar{t})(t \bar{t}) \rightarrow\left(\ell^{ \pm} v_{\ell} b\right)(j j b)\left(\ell^{ \pm \pm} v_{\ell^{\prime}} b\right)(j j b)$. Although the branching fraction for this topology (4.19\%) is smaller than most of the other channels, the presence of two same-sign leptons in the final state allows us to reduce the large QCD background substantially, including that due to the SM production of $t \bar{t} b \bar{b}+$ jets [34]. Therefore, we consider only this channel for our preliminary analysis.

As in the charged Higgs boson case, the heavy Higgs mass can be reconstructed from the signal using the $M_{T 2}$ endpoint technique, and therefore, an additional selection cut on $M_{T 2} \leq M_{h}$ can be used to enhance the signal over the irreducible background, as illustrated in Figure 7 (left). Our simulation results for the predicted number of signal and background events at $\sqrt{s}=14 \mathrm{TeV}$ LHC with $300 \mathrm{fb}^{-1}$ luminosity are shown in Figure 7 (right). From this preliminary analysis, we 

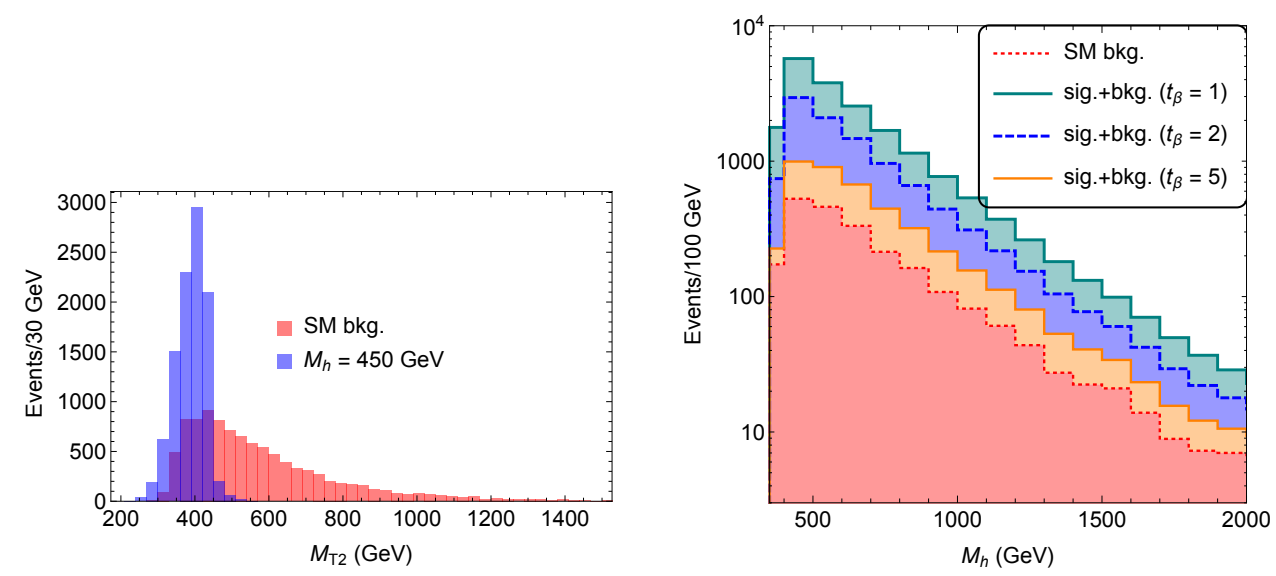

Figure 7: (Left) Heavy neutral Higgs boson mass reconstruction using the $M_{T 2}$ variable. (Right) The four-top signal in the MS-2HDM at $\sqrt{s}=14 \mathrm{TeV}$ LHC with $300 \mathrm{fb}^{-1}$ integrated luminosity.

find that the four-top channel provides the most promising collider signal to probe the heavy neutral Higgs sector in the MS-2HDM for low values of $\tan \beta \lesssim 5$.

The above analysis is also applicable for the CP-odd Higgs boson $a$, which has similar production cross sections and $t \bar{t}$ branching fractions as the CP-even Higgs $h$. However, the $t \bar{t} h(a)$ production cross section as well as the $h(a) \rightarrow t \bar{t}$ branching ratio decreases with increasing $\tan \beta$. This is due to the fact that the $h t \bar{t}$ coupling in the alignment limit is $\cos \alpha / \sin \beta \sim \cot \beta$, which is same as the $a t \bar{t}$ coupling. Thus, the high $\tan \beta$ region of the MS-2HDM cannot be searched via the $t \bar{t} t \bar{t}$ channel proposed above, and one needs to consider the channels involving down-sector Yukawa couplings, e.g. $b \bar{b} b \bar{b}$ and $b \bar{b} \tau^{+} \tau^{-}$. It is also worth commenting here that the simpler process $p p \rightarrow h / a \rightarrow t \bar{t}(b \bar{b})$ at low (high) $\tan \beta$ suffers from a huge SM $t \bar{t}(b \bar{b})$ QCD background, even after imposing an $M_{t \bar{t}(b \bar{b})}$ cut. A combination of kinematic reconstruction and multivariate techniques can be used here to distinguish between the small signal and large background. Some recent studies on extracting the $t \bar{t}$ signal in the context of MSSM have been performed in [35].

\section{Conclusions}

We provide a symmetry justification of the so-called SM alignment limit, independently of the heavy Higgs spectrum and the value of $\tan \beta$ in the 2HDM. We show that there exist only three different symmetry realizations, which could lead to the SM alignment by satisfying the natural alignment condition (2.7) for any value of $\tan \beta$. In the context of the Maximally Symmetric $2 \mathrm{HDM}$ based on the $\mathrm{SO}(5)$ group, we demonstrate how small deviations from this alignment limit are naturally induced by RG effects due to the hypercharge gauge coupling $g^{\prime}$ and third generation Yukawa couplings, which also break the custodial symmetry of the theory. In addition, a non-zero soft $\mathrm{SO}$ (5)-breaking mass parameter is required to yield a viable Higgs spectrum consistent with the existing experimental constraints. Using the LHC Higgs data, which disfavor large deviations from the alignment limit, we derive important constraints on the 2HDM parameter space. In particular, we predict lower limits on the heavy Higgs spectrum, which prevail the present global fit limits in a wide range of parameter space. Depending on the scale where the maximal symmetry could 
be realized in nature, we also obtain an upper limit on the heavy Higgs masses in certain cases, which could be probed during the Run-II phase of the LHC. Finally, we have studied the collider signatures of the heavy Higgs sector beyond the top-quark threshold in the alignment limit. We emphasize that the final states involving third-generation quark final states can become a valuable observational tool to directly probe the heavy Higgs sector of the 2HDM in the alignment limit.

\section{Acknowledgments}

This work of P.S.B.D. and A.P. is supported by the Lancaster-Manchester-Sheffield Consortium for Fundamental Physics under STFC grant ST/L000520/1. P.S.B.D. is also supported in part by a TUM University Foundation Fellowship and the DFG cluster of excellence "Origin and Structure of the Universe".

\section{A. Two-loop RGEs}

The two-loop RGEs for the evolution of the parameters of the general 2HDM potential (1.1) are given in [11], obtained using the generic prescription given in [36], as implemented in the public Mathematica package SARAH [37]. Here we correct some typesetting errors in (B.9)-(B.16) of Ref. [11]. ${ }^{2}$ We clarify that the numerical results presented in [11] remain unchanged, as they were obtained with the correct RGEs, as directly given by the SARAH output.

We start with the two-loop RGEs for the $S U(3)_{c}, S U(2)_{L}$ and $U(1)_{Y}$ gauge couplings:

$$
\begin{aligned}
& \mathscr{D} g_{3}=-\frac{7 g_{3}^{3}}{16 \pi^{2}}+\frac{g_{3}^{3}}{256 \pi^{4}}\left(-26 g_{3}^{2}+\frac{9}{2} g_{2}^{2}+\frac{11}{6} g^{\prime 2}-2 y_{b}^{2}-2 y_{t}^{2}\right), \\
& \mathscr{D} g_{2}=-\frac{3 g_{2}^{3}}{16 \pi^{2}}+\frac{g_{2}^{3}}{256 \pi^{4}}\left(12 g_{3}^{2}+8 g_{2}^{2}+2 g^{\prime 2}-\frac{3}{2} y_{b}^{2}-\frac{3}{2} y_{t}^{2}-\frac{1}{2} y_{\tau}^{2}\right), \\
& \mathscr{D} g^{\prime}=\frac{7 g^{\prime 3}}{16 \pi^{2}}+\frac{g^{\prime 3}}{256 \pi^{4}}\left(\frac{44}{3} g_{3}^{2}+6 g_{2}^{2}+\frac{104}{9} g^{\prime 2}-\frac{5}{6} y_{b}^{2}-\frac{17}{6} y_{t}^{2}-\frac{5}{2} y_{\tau}^{2}\right),
\end{aligned}
$$

where $\mathscr{D} \equiv d / d \ln \mu$ ( $\mu$ being the usual 't-Hooft mass employed in the regularization of ultraviolet divergences in loop integrals), and in the two-loop terms, we have only kept the dominant thirdgeneration contributions.

Similarly for the Yukawa RGEs, we will only consider the third-generation Yukawa couplings, such that for Type-II 2HDM, we have

$$
\begin{aligned}
\mathscr{D} y_{t}= & \frac{y_{t}}{16 \pi^{2}}\left(-8 g_{3}^{2}-\frac{9}{4} g_{2}^{2}-\frac{17}{12} g^{\prime 2}+\frac{9}{2} y_{t}^{2}+\frac{1}{2} y_{b}^{2}\right) \\
& +\frac{y_{t}}{256 \pi^{4}}\left[-108 g_{3}^{4}-\frac{21}{4} g_{2}^{4}+\frac{1267}{216} g^{\prime 4}+9 g_{2}^{2} g_{3}^{2}-\frac{3}{4} g_{2}^{2} g^{\prime 2}+\frac{19}{9} g_{3}^{2} g^{\prime 2}+6 \lambda_{2}^{2}+\lambda_{3}^{2}\right. \\
& +\lambda_{3} \lambda_{4}+\lambda_{4}^{2}+\frac{3}{2}\left(\lambda_{5}^{2}+\lambda_{6}^{2}+3 \lambda_{7}^{2}\right)+\left(\frac{16}{3} g_{3}^{2}+\frac{33}{16} g_{2}^{2}-\frac{41}{144} g^{\prime 2}-2 \lambda_{3}+2 \lambda_{4}\right) y_{b}^{2} \\
& \left.+\left(36 g_{3}^{2}+\frac{225}{16} g_{2}^{2}+\frac{131}{16} g^{\prime 2}-12 \lambda_{2}\right) y_{t}^{2}-\frac{5}{2} y_{b}^{4}-12 y_{t}^{4}-\frac{5}{2} y_{b}^{2} y_{t}^{2}-\frac{3}{4} y_{b}^{2} y_{\tau}^{2}\right],
\end{aligned}
$$

\footnotetext{
${ }^{2}$ We thank Gabriel Lee and Carlos Wagner for pointing these out and for carefully checking our expressions against theirs [38].
} 


$$
\begin{aligned}
\mathscr{D} y_{b}= & \frac{y_{b}}{16 \pi^{2}}\left(-8 g_{3}^{2}-\frac{9}{4} g_{2}^{2}-\frac{5}{12} g^{\prime 2}+\frac{9}{2} y_{b}^{2}+\frac{1}{2} y_{t}^{2}+y_{\tau}^{2}\right) \\
& +\frac{y_{b}}{256 \pi^{4}}\left[-108 g_{3}^{4}-\frac{21}{4} g_{2}^{4}-\frac{113}{216} g^{\prime 4}+9 g_{2}^{2} g_{3}^{2}-\frac{9}{4} g_{2}^{2} g^{2}+\frac{31}{9} g_{3}^{2} g^{\prime 2}+6 \lambda_{1}^{2}+\lambda_{3}^{2}\right. \\
& +\lambda_{3} \lambda_{4}+\lambda_{4}^{2}+\frac{3}{2}\left(\lambda_{5}^{2}+3 \lambda_{6}^{2}+\lambda_{7}^{2}\right)+\left(\frac{16}{3} g_{3}^{2}+\frac{33}{16} g_{2}^{2}-\frac{53}{144} g^{\prime 2}-2 \lambda_{3}+2 \lambda_{4}\right) y_{t}^{2} \\
& +\left(\frac{15}{8} g_{2}^{2}+\frac{25}{8} g^{\prime 2}\right) y_{\tau}^{2}+\left(36 g_{3}^{2}+\frac{225}{16} g_{2}^{2}+\frac{79}{16} g^{\prime 2}-12 \lambda_{1}\right) y_{b}^{2} \\
& \left.-12 y_{b}^{4}-\frac{9}{4} y_{\tau}^{4}-\frac{5}{2} y_{t}^{4}-\frac{5}{2} y_{b}^{2} y_{t}^{2}-\frac{9}{4} y_{b}^{2} y_{\tau}^{2}\right] \\
\mathscr{D} y_{\tau}= & \frac{y_{\tau}}{16 \pi^{2}}\left(-\frac{9}{4} g_{2}^{2}-\frac{15}{4} g^{\prime 2}+3 y_{b}^{2}+\frac{5}{2} y_{\tau}^{2}\right) \\
& +\frac{y_{\tau}}{256 \pi^{4}}\left[-\frac{21}{4} g_{2}^{4}+\frac{161}{8} g^{\prime 4}+\frac{9}{4} g_{2}^{2} g^{\prime 2}+6 \lambda_{1}^{2}+\lambda_{3}^{2}+\lambda_{3} \lambda_{4}+\lambda_{4}^{2}\right. \\
& +\frac{3}{2}\left(\lambda_{5}^{2}+3 \lambda_{6}^{2}+\lambda_{7}^{2}\right)+\left(20 g_{3}^{2}+\frac{45}{8} g_{2}^{2}+\frac{25}{24} g^{\prime 2}\right) y_{b}^{2} \\
& \left.+\left(\frac{165}{16} g_{2}^{2}+\frac{179}{16} g^{\prime 2}-12 \lambda_{1}\right) y_{\tau}^{2}-\frac{27}{4} y_{b}^{4}-3 y_{\tau}^{4}-\frac{27}{4} y_{b}^{2} y_{\tau}^{2}-\frac{9}{4} y_{b}^{2} y_{t}^{2}\right] .
\end{aligned}
$$

Similarly, the two-loop RGEs for the VEVs are given by

$$
\begin{aligned}
\mathscr{D} v_{1}= & \frac{v_{1}}{16 \pi^{2}}\left[\frac{3}{4}\left(3 g_{2}^{2}+g^{\prime 2}\right)-3 y_{b}^{2}-y_{\tau}^{2}\right] \\
& +\frac{v_{1}}{256 \pi^{4}}\left[\frac{435}{32} g_{2}^{4}-\frac{149}{32} g^{\prime 4}-\frac{3}{16} g_{2}^{2} g^{\prime 2}-6 \lambda_{1}^{2}-\lambda_{3}^{2}-\lambda_{3} \lambda_{4}-\lambda_{4}^{2}\right. \\
& -\frac{3}{2}\left(\lambda_{5}^{2}+3 \lambda_{6}^{2}+\lambda_{7}^{2}\right)-\left(20 g_{3}^{2}+\frac{45}{8} g_{2}^{2}+\frac{25}{24} g^{\prime 2}\right) y_{b}^{2}-\left(\frac{15}{8} g_{2}^{2}+\frac{25}{8} g^{\prime 2}\right) y_{\tau}^{2} \\
& \left.+\frac{27}{4} y_{b}^{4}+\frac{9}{4} y_{\tau}^{4}+\frac{9}{4} y_{b}^{2} y_{t}^{2}\right]-\frac{3 v_{2}}{512 \pi^{4}}\left[\left(2 \lambda_{1}+\lambda_{345}\right) \lambda_{6}+\left(2 \lambda_{2}+\lambda_{345}\right) \lambda_{7}\right], \\
\mathscr{D} v_{2}= & \frac{v_{2}}{16 \pi^{2}}\left[\frac{3}{4}\left(3 g_{2}^{2}+g^{\prime 2}\right)-3 y_{t}^{2}\right] \\
& +\frac{v_{2}}{256 \pi^{4}}\left[\frac{435}{32} g_{2}^{4}-\frac{149}{32} g^{\prime 4}-\frac{3}{16} g_{2}^{2} g^{\prime 2}-6 \lambda_{2}^{2}-\lambda_{3}^{2}-\lambda_{3} \lambda_{4}-\lambda_{4}^{2}\right. \\
& \left.-\frac{3}{2}\left(\lambda_{5}^{2}+\lambda_{6}^{2}+3 \lambda_{7}^{2}\right)-\left(20 g_{3}^{2}+\frac{45}{8} g_{2}^{2}+\frac{85}{24} g^{\prime 2}\right) y_{t}^{2}+\frac{9}{4} y_{b}^{2} y_{t}^{2}+\frac{27}{4} y_{t}^{4}\right] \\
& -\frac{3 v_{1}}{512 \pi^{4}}\left[\left(2 \lambda_{1}+\lambda_{345}\right) \lambda_{6}+\left(2 \lambda_{2}+\lambda_{345}\right) \lambda_{7}\right] .
\end{aligned}
$$

The two-loop RGEs for all the scalar quartic couplings appearing in (1.1) in the Type-II 2HDM are given by

$$
\begin{gathered}
\mathscr{D} \lambda_{1}=\frac{1}{16 \pi^{2}}\left[\frac{3}{8}\left(3 g_{2}^{4}+g^{\prime 4}+2 g_{2}^{2} g^{\prime 2}\right)-3 \lambda_{1}\left(3 g_{2}^{2}+g^{\prime 2}\right)+24 \lambda_{1}^{2}+2 \lambda_{3}^{2}+2 \lambda_{3} \lambda_{4}+\lambda_{4}^{2}\right. \\
\left.+\lambda_{5}^{2}+12 \lambda_{6}^{2}+4 \lambda_{1}\left(3 y_{b}^{2}+y_{\tau}^{2}\right)-6 y_{b}^{4}-2 y_{\tau}^{4}\right]
\end{gathered}
$$




$$
\begin{aligned}
& +\frac{1}{256 \pi^{4}}\left[\frac{1}{16}\left(291 g_{2}^{6}-101 g_{2}^{4} g^{\prime 2}-191 g_{2}^{2} g^{\prime 4}-131 g^{\prime 6}\right)\right. \\
& -\frac{1}{8}\left(51 g_{2}^{4}-78 g_{2}^{2} g^{\prime 2}-217 g^{\prime 4}\right) \lambda_{1}+\frac{5}{2}\left(3 g_{2}^{4}+g^{\prime 4}\right) \lambda_{3}+\frac{5}{4}\left(3 g_{2}^{4}+2 g_{2}^{2} g^{\prime 2}+g^{\prime 4}\right) \lambda_{4} \\
& +\left(3 g_{2}^{2}+g^{\prime 2}\right)\left(36 \lambda_{1}^{2}+4 \lambda_{3}^{2}+4 \lambda_{3} \lambda_{4}+\lambda_{4}^{2}+18 \lambda_{6}^{2}\right)+g^{\prime 2}\left(\lambda_{4}^{2}-\lambda_{5}^{2}\right)-312 \lambda_{1}^{3} \\
& -8 \lambda_{3}^{3}-6 \lambda_{4}^{3}-20 \lambda_{1} \lambda_{3} \lambda_{4}-4\left(5 \lambda_{1}+3 \lambda_{4}\right) \lambda_{3}^{2}-4\left(3 \lambda_{1}+4 \lambda_{3}\right) \lambda_{4}^{2} \\
& -2\left(7 \lambda_{1}+10 \lambda_{3}+11 \lambda_{4}\right) \lambda_{5}^{2}-2\left(159 \lambda_{1}+33 \lambda_{3}+35 \lambda_{4}+37 \lambda_{5}\right) \lambda_{6}^{2} \\
& -4\left(9 \lambda_{3}+7 \lambda_{4}+5 \lambda_{5}\right) \lambda_{6} \lambda_{7}+2\left(3 \lambda_{1}-9 \lambda_{3}-7 \lambda_{4}-5 \lambda_{5}\right) \lambda_{7}^{2} \\
& -\left\{\frac{9}{4} g_{2}^{4}-\frac{9}{2} g_{2}^{2} g^{\prime 2}-\frac{5}{4} g^{\prime 4}-\left(\frac{45}{2} g_{2}^{2}+80 g_{3}^{2}+\frac{25}{6} g^{\prime 2}\right) \lambda_{1}+36\left(4 \lambda_{1}^{2}+\lambda_{6}^{2}\right)\right\} y_{b}^{2} \\
& -\left(32 g_{3}^{2}-\frac{4}{3} g^{2}+3 \lambda_{1}\right) y_{b}^{4}-6\left(2 \lambda_{3}^{2}+2 \lambda_{3} \lambda_{4}+\lambda_{4}^{2}+\lambda_{5}^{2}+6 \lambda_{6}^{2}\right) y_{t}^{2} \\
& -\left\{\frac{3}{4} g_{2}^{4}-\frac{11}{2} g_{2}^{2} g^{\prime 2}+\frac{25}{4} g^{\prime 4}-\frac{5}{2}\left(3 g_{2}^{2}+5 g^{\prime 2}\right) \lambda_{1}+12\left(4 \lambda_{1}^{2}+\lambda_{6}^{2}\right)\right\} y_{\tau}^{2} \\
& \left.-\left(4 g^{\prime 2}+\lambda_{1}\right) y_{\tau}^{4}-9 \lambda_{1} y_{b}^{2} y_{t}^{2}+6 y_{t}^{2} y_{b}^{4}+30 y_{b}^{6}+10 y_{\tau}^{6}\right] \text {, } \\
& \mathscr{D} \lambda_{2}=\frac{1}{16 \pi^{2}}\left[\frac{3}{8}\left(3 g_{2}^{4}+g^{\prime 4}+2 g_{2}^{2} g^{2}\right)-3 \lambda_{2}\left(3 g_{2}^{2}+g^{\prime 2}\right)+24 \lambda_{2}^{2}+2 \lambda_{3}^{2}+2 \lambda_{3} \lambda_{4}\right. \\
& \left.+\lambda_{4}^{2}+\lambda_{5}^{2}+12 \lambda_{7}^{2}+12 \lambda_{2} y_{t}^{2}-6 y_{t}^{4}\right] \\
& +\frac{1}{256 \pi^{4}}\left[\frac{1}{16}\left(291 g_{2}^{6}-101 g_{2}^{4} g^{\prime 2}-191 g_{2}^{2} g^{\prime 4}-131 g^{\prime 6}\right)\right. \\
& -\frac{1}{8}\left(51 g_{2}^{4}-78 g_{2}^{2} g^{\prime 2}-217 g^{\prime 4}\right) \lambda_{2}+\frac{5}{2}\left(3 g_{2}^{4}+g^{\prime 4}\right) \lambda_{3}+\frac{5}{4}\left(3 g_{2}^{4}+2 g_{2}^{2} g^{\prime 2}+g^{\prime 4}\right) \lambda_{4} \\
& +\left(3 g_{2}^{2}+g^{\prime 2}\right)\left(36 \lambda_{2}^{2}+4 \lambda_{3}^{2}+4 \lambda_{3} \lambda_{4}+\lambda_{4}^{2}+18 \lambda_{7}^{2}\right)+g^{\prime 2}\left(\lambda_{4}^{2}-\lambda_{5}^{2}\right)-312 \lambda_{2}^{3} \\
& -8 \lambda_{3}^{3}-6 \lambda_{4}^{3}-20 \lambda_{2} \lambda_{3} \lambda_{4}-4\left(5 \lambda_{2}+3 \lambda_{4}\right) \lambda_{3}^{2}-4\left(3 \lambda_{2}+4 \lambda_{3}\right) \lambda_{4}^{2} \\
& -2\left(7 \lambda_{2}+10 \lambda_{3}+11 \lambda_{4}\right) \lambda_{5}^{2}+2\left(3 \lambda_{2}-9 \lambda_{3}-7 \lambda_{4}-5 \lambda_{5}\right) \lambda_{6}^{2} \\
& -4\left(9 \lambda_{3}+7 \lambda_{4}+5 \lambda_{5}\right) \lambda_{6} \lambda_{7}-2\left(159 \lambda_{2}+33 \lambda_{3}+35 \lambda_{4}+37 \lambda_{5}\right) \lambda_{7}^{2} \\
& -6\left(2 \lambda_{3}^{2}+2 \lambda_{3} \lambda_{4}+\lambda_{4}^{2}+\lambda_{5}^{2}+6 \lambda_{7}^{2}\right) y_{b}^{2}-\left\{\frac{9}{4} g_{2}^{4}-\frac{21}{2} g_{2}^{2} g^{2}+\frac{19}{4} g^{\prime 4}\right. \\
& \left.-\left(\frac{45}{2} g_{2}^{2}+80 g_{3}^{2}+\frac{85}{6} g^{\prime 2}\right) \lambda_{2}+36\left(4 \lambda_{2}^{2}+\lambda_{7}^{2}\right)\right\} y_{t}^{2}-9 \lambda_{2} y_{b}^{2} y_{t}^{2}+6 y_{b}^{2} y_{t}^{4} \\
& \left.-\left(32 g_{3}^{2}+\frac{8}{3} g^{\prime 2}+3 \lambda_{2}\right) y_{t}^{4}+30 y_{t}^{6}-2\left(2 \lambda_{3}^{2}+2 \lambda_{3} \lambda_{4}+\lambda_{4}^{2}+\lambda_{5}^{2}+6 \lambda_{7}^{2}\right) y_{\tau}^{2}\right] \text {, } \\
& \mathscr{D} \lambda_{3}=\frac{1}{16 \pi^{2}}\left[\frac{3}{4}\left(3 g_{2}^{4}+g^{\prime 4}-2 g_{2}^{2} g^{\prime 2}\right)-3 \lambda_{3}\left(3 g_{2}^{2}+g^{2}\right)+4\left(\lambda_{1}+\lambda_{2}\right)\left(3 \lambda_{3}+\lambda_{4}\right)+4 \lambda_{3}^{2}\right. \\
& \left.+2\left(\lambda_{4}^{2}+\lambda_{5}^{2}\right)+4\left(\lambda_{6}^{2}+\lambda_{7}^{2}+4 \lambda_{6} \lambda_{7}\right)+2 \lambda_{3}\left(3 y_{b}^{2}+y_{\tau}^{2}+3 y_{t}^{2}\right)-12 y_{b}^{2} y_{t}^{2}\right]
\end{aligned}
$$




$$
\begin{aligned}
& +\frac{1}{256 \pi^{4}}\left[\frac{1}{8}\left(291 g_{2}^{6}+11 g_{2}^{4} g^{\prime 2}+101 g_{2}^{2} g^{\prime 4}-131 g^{\prime 6}\right)\right. \\
& +\frac{5}{2}\left(9 g_{2}^{4}-2 g_{2}^{2} g^{\prime 2}+3 g^{\prime 4}\right)\left(\lambda_{1}+\lambda_{2}\right)-\frac{1}{8}\left(111 g_{2}^{4}-22 g_{2}^{2} g^{\prime 2}-197 g^{\prime 4}\right) \lambda_{3} \\
& +2\left(3 g_{2}^{2}+g^{\prime 2}\right)\left[12\left(\lambda_{1}+\lambda_{2}\right) \lambda_{3}+\lambda_{3}^{2}+\lambda_{4}^{2}\right]-4\left(\lambda_{1}^{2}+\lambda_{2}^{2}\right)\left(15 \lambda_{3}+4 \lambda_{4}\right) \\
& -4\left(\lambda_{1}+\lambda_{2}\right)\left(18 \lambda_{3}^{2}+7 \lambda_{4}^{2}+8 \lambda_{3} \lambda_{4}+9 \lambda_{5}^{2}\right)-12\left(\lambda_{3}^{3}+\lambda_{4}^{3}+g_{2}^{2} \lambda_{3} \lambda_{4}\right) \\
& +\left(\frac{15}{2} g_{2}^{4}-3 g_{2}^{2} g^{\prime 2}+\frac{5}{2} g^{\prime 4}\right) \lambda_{4}+4\left(9 g_{2}^{2}+2 g^{\prime 2}\right)\left(\lambda_{1}+\lambda_{2}\right) \lambda_{4} \\
& -4 \lambda_{3} \lambda_{4}\left(\lambda_{3}+4 \lambda_{4}\right)-2\left(9 \lambda_{3}+22 \lambda_{4}\right) \lambda_{5}^{2}-4 g^{\prime 2}\left(\lambda_{4}^{2}-\lambda_{5}^{2}\right)+2 g^{\prime 2}\left(\lambda_{6}^{2}+\lambda_{7}^{2}\right) \\
& -4\left(31 \lambda_{1}+11 \lambda_{2}\right) \lambda_{6}^{2}-4\left(15 \lambda_{3}+17 \lambda_{4}+17 \lambda_{5}\right)\left(\lambda_{6}^{2}+\lambda_{7}^{2}\right)+4\left(27 g_{2}^{2}+8 g^{2}\right) \lambda_{6} \lambda_{7} \\
& -8\left[11\left(\lambda_{1}+\lambda_{2}+2 \lambda_{3}+\lambda_{4}\right)+9 \lambda_{5}\right] \lambda_{6} \lambda_{7}-4\left(11 \lambda_{1}+31 \lambda_{2}\right) \lambda_{7}^{2} \\
& -\left\{\frac{1}{4}\left(9 g_{2}^{4}-5 g^{\prime 4}+18 g_{2}^{2} g^{\prime 2}\right)-\left(40 g_{3}^{2}+\frac{45}{4} g_{2}^{2}+\frac{25}{12} g^{\prime 2}\right) \lambda_{3}\right\} y_{b}^{2} \\
& -\frac{1}{4}\left\{3 g_{2}^{4}+25 g^{\prime 4}+22 g_{2}^{2} g^{\prime 2}-5\left(3 g_{2}^{2}+5 g^{\prime 2}\right) \lambda_{3}\right\} y_{\tau}^{2} \\
& -2\left\{2 \lambda_{3}^{2}+\lambda_{4}^{2}+4 \lambda_{1}\left(3 \lambda_{3}+\lambda_{4}\right)+\lambda_{5}^{2}+4 \lambda_{6}^{2}+8 \lambda_{6} \lambda_{7}\right\}\left(3 y_{b}^{2}+y_{\tau}^{2}\right) \\
& -\left\{\frac{9}{4} g_{2}^{4}+\frac{21}{2} g_{2}^{2} g^{\prime 2}+\frac{19}{4} g^{\prime 4}-\left(40 g_{3}^{2}+\frac{45}{4} g_{2}^{2}+\frac{85}{12} g^{\prime 2}\right) \lambda_{3}\right. \\
& \left.+6\left(12 \lambda_{2} \lambda_{3}+2 \lambda_{3}^{2}+4 \lambda_{2} \lambda_{4}+\lambda_{4}^{2}+\lambda_{5}^{2}+8 \lambda_{6} \lambda_{7}+4 \lambda_{7}^{2}\right)\right\} y_{t}^{2} \\
& \left.-\left(64 g_{3}^{2}+\frac{4}{3} g^{\prime 2}-15 \lambda_{3}\right) y_{b}^{2} y_{t}^{2}+36 y_{b}^{4} y_{t}^{2}-\frac{9}{2} \lambda_{3}\left[3\left(y_{b}^{4}+y_{t}^{4}\right)+y_{\tau}^{4}\right]+36 y_{b}^{2} y_{t}^{4}\right] \text {, } \\
& \mathscr{D} \lambda_{4}=\frac{1}{16 \pi^{2}}\left[3 g_{2}^{2} g^{\prime 2}-3 \lambda_{4}\left(3 g_{2}^{2}+g^{\prime 2}\right)+4\left(\lambda_{1}+\lambda_{2}+2 \lambda_{3}\right) \lambda_{4}+4 \lambda_{4}^{2}+8 \lambda_{5}^{2}\right. \\
& \left.+10\left(\lambda_{6}^{2}+\lambda_{7}^{2}\right)+4 \lambda_{6} \lambda_{7}+2 \lambda_{4}\left\{3\left(y_{b}^{2}+y_{t}^{2}\right)+y_{\tau}^{2}\right\}+12 y_{b}^{2} y_{t}^{2}\right] \\
& +\frac{1}{256 \pi^{4}}\left[-14 g_{2}^{4} g^{\prime 2}-\frac{73}{2} g_{2}^{2} g^{\prime 4}+2 g_{2}^{2} g^{\prime 2}\left\{5\left(\lambda_{1}+\lambda_{2}\right)+\lambda_{3}\right\}\right. \\
& -\frac{1}{8}\left(231 g_{2}^{4}-102 g_{2}^{2} g^{\prime 2}-157 g^{\prime 4}\right) \lambda_{4}+4\left\{2 g^{\prime 2}\left(\lambda_{1}+\lambda_{2}\right)-7\left(\lambda_{1}^{2}+\lambda_{2}^{2}+\lambda_{3}^{2}\right)\right\} \lambda_{4} \\
& +4\left(9 g_{2}^{2}+g^{\prime 2}\right) \lambda_{3} \lambda_{4}-40\left(\lambda_{1}+\lambda_{2}\right)\left(2 \lambda_{3} \lambda_{4}+\lambda_{4}^{2}\right)+2\left(9 g_{2}^{2}+4 g^{\prime 2}\right) \lambda_{4}^{2}-28 \lambda_{3} \lambda_{4}^{2} \\
& +2\left(27 g_{2}^{2}+8 g^{\prime 2}\right) \lambda_{5}^{2}-48\left(\lambda_{1}+\lambda_{2}+\lambda_{3}\right) \lambda_{5}^{2}-26 \lambda_{4} \lambda_{5}^{2}+8 g^{\prime 2} \lambda_{6} \lambda_{7} \\
& +2\left\{27 g_{2}^{2}+7 g^{\prime 2}-2\left(18 \lambda_{3}+17 \lambda_{4}+20 \lambda_{5}\right)\right\}\left(\lambda_{6}^{2}+\lambda_{7}^{2}\right)-4\left(37 \lambda_{1}+5 \lambda_{2}\right) \lambda_{6}^{2} \\
& -8\left\{5\left(\lambda_{1}+\lambda_{2}+2 \lambda_{3}+4 \lambda_{4}\right)+12 \lambda_{5}\right\} \lambda_{6} \lambda_{7}-4\left(5 \lambda_{1}+37 \lambda_{2}\right) \lambda_{7}^{2} \\
& +\left\{9 g_{2}^{2} g^{\prime 2}+\left(40 g_{3}^{2}+\frac{45}{4} g_{2}^{2}+\frac{25}{12} g^{\prime 2}\right) \lambda_{4}-12\left[2\left(\lambda_{1}+\lambda_{3}\right) \lambda_{4}+\lambda_{4}^{2}+2 \lambda_{5}^{2}\right.\right. \\
& \left.\left.+5 \lambda_{6}^{2}+\lambda_{6} \lambda_{7}\right]\right\} y_{b}^{2}+\left\{21 g_{2}^{2} g^{\prime 2}+\left(40 g_{3}^{2}+\frac{45}{4} g_{2}^{2}+\frac{85}{12} g^{\prime 2}\right) \lambda_{4}-12\left[2\left(\lambda_{2}+\lambda_{3}\right) \lambda_{4}\right.\right. \\
& \left.\left.+\lambda_{4}^{2}+2 \lambda_{5}^{2}+\lambda_{6} \lambda_{7}+5 \lambda_{7}^{2}\right]\right\} y_{t}^{2}+\left(64 g_{3}^{2}+\frac{4}{3} g^{\prime 2}-24 \lambda_{3}-33 \lambda_{4}\right) y_{b}^{2} y_{t}^{2}
\end{aligned}
$$




$$
\begin{aligned}
& +\left\{11 g_{2}^{2} g^{\prime 2}+\frac{5}{4}\left(3 g_{2}^{2}+5 g^{\prime 2}\right) \lambda_{4}-4\left[2\left(\lambda_{1}+\lambda_{3}\right) \lambda_{4}+\lambda_{4}^{2}+2 \lambda_{5}^{2}\right.\right. \\
& \left.\left.\left.+5 \lambda_{6}^{2}+\lambda_{6} \lambda_{7}\right]\right\} y_{\tau}^{2}-\frac{9}{2} \lambda_{4}\left\{3\left(y_{b}^{4}+y_{t}^{4}\right)+y_{\tau}^{4}\right\}-24 y_{b}^{2} y_{t}^{2}\left(y_{b}^{2}+y_{t}^{2}\right)\right], \\
& \mathscr{D} \lambda_{5}=\frac{1}{16 \pi^{2}}\left[-3 \lambda_{5}\left(3 g_{2}^{2}+g^{\prime 2}\right)+4\left(\lambda_{1}+\lambda_{2}+2 \lambda_{3}+3 \lambda_{4}\right) \lambda_{5}+10\left(\lambda_{6}^{2}+\lambda_{7}^{2}\right)\right. \\
& \left.+4 \lambda_{6} \lambda_{7}+2 \lambda_{5}\left\{3\left(y_{b}^{2}+y_{t}^{2}\right)+y_{\tau}^{2}\right\}\right] \\
& +\frac{1}{256 \pi^{4}}\left[-\frac{1}{8}\left(231 g_{2}^{4}-38 g_{2}^{2} g^{\prime 2}-157 g^{\prime 4}\right) \lambda_{5}+4\left(9 g_{2}^{2}+4 g^{\prime 2}\right) \lambda_{3} \lambda_{5}\right. \\
& -4\left\{g^{\prime 2}\left(\lambda_{1}+\lambda_{2}\right)+7\left(\lambda_{1}^{2}+\lambda_{2}^{2}+\lambda_{3}^{2}\right)\right\} \lambda_{5}-8\left(\lambda_{1}+\lambda_{2}\right)\left(10 \lambda_{3}+11 \lambda_{4}\right) \lambda_{5} \\
& +4\left\{6\left(3 g_{2}^{2}+g^{\prime 2}\right)-19 \lambda_{3}-8 \lambda_{4}\right\} \lambda_{4} \lambda_{5}+6 \lambda_{5}^{3}-4\left(37 \lambda_{1}+5 \lambda_{2}\right) \lambda_{6}^{2} \\
& -4\left(5 \lambda_{1}+37 \lambda_{2}\right) \lambda_{7}^{2}+2\left\{27 g_{2}^{2}+10 g^{\prime 2}-2\left(18 \lambda_{3}+19 \lambda_{4}+18 \lambda_{5}\right)\right\}\left(\lambda_{6}^{2}+\lambda_{7}^{2}\right) \\
& -4\left\{g^{\prime 2}+10\left(\lambda_{1}+\lambda_{2}+2 \lambda_{3}\right)+22 \lambda_{4}+42 \lambda_{5}\right\} \lambda_{6} \lambda_{7} \\
& +\left\{\left(40 g_{3}^{2}+\frac{45}{4} g_{2}^{2}+\frac{25}{12} g^{\prime 2}\right) \lambda_{5}-12\left[2\left(\lambda_{1}+\lambda_{3}\right) \lambda_{5}+3 \lambda_{4} \lambda_{5}+5 \lambda_{6}^{2}+\lambda_{6} \lambda_{7}\right]\right\} y_{b}^{2} \\
& +\left\{\left(40 g_{3}^{2}+\frac{45}{4} g_{2}^{2}+\frac{85}{12} g^{\prime 2}\right) \lambda_{5}-12\left[2\left(\lambda_{2}+\lambda_{3}\right) \lambda_{5}+3 \lambda_{4} \lambda_{5}+\lambda_{6} \lambda_{7}+5 \lambda_{7}^{2}\right\} y_{t}^{2}\right. \\
& +\left\{\left(\frac{15}{4} g_{2}^{2}+\frac{25}{4} g^{\prime 2}\right) \lambda_{5}-8\left(\lambda_{1}+\lambda_{3}\right) \lambda_{5}-12 \lambda_{4} \lambda_{5}-20 \lambda_{6}^{2}-4 \lambda_{6} \lambda_{7}\right\} y_{\tau}^{2} \\
& \left.-\frac{1}{2} \lambda_{5}\left\{3\left(y_{b}^{4}+y_{t}^{4}\right)+y_{\tau}^{4}\right\}-33 \lambda_{5} y_{b}^{2} y_{t}^{2}\right] \text {, } \\
& \mathscr{D} \lambda_{6}=\frac{1}{16 \pi^{2}}\left[-3 \lambda_{6}\left(3 g_{2}^{2}+g^{\prime 2}\right)+2\left(12 \lambda_{1}+3 \lambda_{3}+4 \lambda_{4}\right) \lambda_{6}+2\left(3 \lambda_{3}+2 \lambda_{4}\right) \lambda_{7}\right. \\
& \left.+10 \lambda_{5} \lambda_{6}+2 \lambda_{5} \lambda_{7}+3 \lambda_{6}\left(3 y_{b}^{2}+y_{t}^{2}+y_{\tau}^{2}\right)\right] \\
& +\frac{1}{256 \pi^{4}}\left[-\frac{1}{8}\left(141 g_{2}^{4}-58 g_{2}^{2} g^{\prime 2}-187 g^{\prime 4}\right) \lambda_{6}+6\left(3 g_{2}^{2}+g^{\prime 2}\right)\left(6 \lambda_{1}+\lambda_{3}\right) \lambda_{6}\right. \\
& -6\left(53 \lambda_{1}^{2}-\lambda_{2}^{2}\right) \lambda_{6}-4\left(33 \lambda_{1}+9 \lambda_{2}+8 \lambda_{3}\right) \lambda_{3} \lambda_{6}+2\left(18 g_{2}^{2}+5 g^{2}\right) \lambda_{4} \lambda_{6} \\
& -2\left(70 \lambda_{1}+14 \lambda_{2}+34 \lambda_{3}+17 \lambda_{4}\right) \lambda_{4} \lambda_{6}+2\left(27 g_{2}^{2}+10 g^{\prime 2}\right) \lambda_{5} \lambda_{6} \\
& -4\left(37 \lambda_{1}+5 \lambda_{2}+18 \lambda_{3}+19 \lambda_{4}+9 \lambda_{5}\right) \lambda_{5} \lambda_{6}-111 \lambda_{6}^{3}-42 \lambda_{7}^{3} \\
& +\frac{5}{4}\left(9 g_{2}^{4}+2 g_{2}^{2} g^{\prime 2}+3 g^{\prime 4}\right) \lambda_{7}+12\left(3 g_{2}^{2}+g^{\prime 2}\right) \lambda_{3} \lambda_{7}-36\left(\lambda_{1}+\lambda_{2}+\lambda_{3}\right) \lambda_{3} \lambda_{7} \\
& +2\left(9 g_{2}^{2}+4 g^{2}\right) \lambda_{4} \lambda_{7}-2\left\{14\left(\lambda_{1}+\lambda_{2}+2 \lambda_{3}\right)+17 \lambda_{4}\right\} \lambda_{4} \lambda_{7} \\
& -2\left\{g^{\prime 2}+10\left(\lambda_{1}+\lambda_{2}+2 \lambda_{3}\right)+22 \lambda_{4}+21 \lambda_{5}\right\} \lambda_{5} \lambda_{7}-3\left(42 \lambda_{6}+11 \lambda_{7}\right) \lambda_{6} \lambda_{7} \\
& +\left\{60 g_{3}^{2}+\frac{135}{8} g_{2}^{2}+\frac{25}{8} g^{\prime 2}-6\left(24 \lambda_{1}+3 \lambda_{3}+4 \lambda_{4}+5 \lambda_{5}\right)\right\} \lambda_{6} y_{b}^{2}
\end{aligned}
$$




$$
\begin{aligned}
& +\left\{20 g_{3}^{2}+\frac{45}{8} g_{2}^{2}+\frac{85}{24} g^{\prime 2}-6\left(3 \lambda_{3}+4 \lambda_{4}+5 \lambda_{5}\right)\right\} \lambda_{6} y_{t}^{2} \\
& +\left\{\frac{15}{8}\left(3 g_{2}^{2}+5 g^{\prime 2}\right)-2\left(24 \lambda_{1}+3 \lambda_{3}+4 \lambda_{4}+5 \lambda_{5}\right)\right\} \lambda_{6} y_{\tau}^{2} \\
& \left.-12\left(3 \lambda_{3}+2 \lambda_{4}+\lambda_{5}\right) \lambda_{7} y_{t}^{2}-\frac{1}{4}\left(27 y_{t}^{4}+33 y_{b}^{4}+11 y_{\tau}^{4}\right) \lambda_{6}-21 \lambda_{6} y_{b}^{2} y_{t}^{2}\right], \\
& \mathscr{D} \lambda_{7}=\frac{1}{16 \pi^{2}}\left[-3 \lambda_{7}\left(3 g_{2}^{2}+g^{\prime 2}\right)+2\left(12 \lambda_{2}+3 \lambda_{3}+4 \lambda_{4}\right) \lambda_{7}+2\left(3 \lambda_{3}+2 \lambda_{4}\right) \lambda_{6}\right. \\
& \left.+10 \lambda_{5} \lambda_{7}+2 \lambda_{5} \lambda_{6}+\lambda_{7}\left(3 y_{b}^{2}+9 y_{t}^{2}+y_{\tau}^{2}\right)\right] \\
& +\frac{1}{256 \pi^{4}}\left[\frac{5}{4}\left(9 g_{2}^{4}+2 g_{2}^{2} g^{\prime 2}+3 g^{\prime 4}\right) \lambda_{6}+12\left(3 g_{2}^{2}+g^{\prime 2}\right) \lambda_{3} \lambda_{6}\right. \\
& -36\left(\lambda_{1}+\lambda_{2}+\lambda_{3}\right) \lambda_{3} \lambda_{6}+2\left(9 g_{2}^{2}+4 g^{2}\right) \lambda_{4} \lambda_{6}-28\left(\lambda_{1}+\lambda_{2}+2 \lambda_{3}\right) \lambda_{4} \lambda_{6} \\
& -34 \lambda_{4}^{2} \lambda_{6}-2 g^{\prime 2} \lambda_{5} \lambda_{6}-4\left\{5\left(\lambda_{1}+\lambda_{2}+2 \lambda_{3}\right)+11 \lambda_{4}\right\} \lambda_{5} \lambda_{6}-42\left(\lambda_{5}^{2}+\lambda_{6}^{2}\right) \lambda_{6} \\
& -\frac{1}{8}\left(141 g_{2}^{4}-58 g_{2}^{2} g^{\prime 2}-187 g^{\prime 4}\right) \lambda_{7}+6 \lambda_{1}^{2} \lambda_{7}+36\left(3 g_{2}^{2}+g^{\prime 2}\right) \lambda_{2} \lambda_{7}-318 \lambda_{2}^{2} \lambda_{7} \\
& +6\left(3 g_{2}^{2}+g^{2}\right) \lambda_{3} \lambda_{7}-12\left(3 \lambda_{1}+11 \lambda_{2}\right) \lambda_{3} \lambda_{7}-32 \lambda_{3}^{2} \lambda_{7}+2\left(18 g_{2}^{2}+5 g^{\prime 2}\right) \lambda_{4} \lambda_{7} \\
& -4\left(7 \lambda_{1}+35 \lambda_{2}+17 \lambda_{3}\right) \lambda_{4} \lambda_{7}-34 \lambda_{4}^{2} \lambda_{7}+2\left(27 g_{2}^{2}+10 g^{2}\right) \lambda_{5} \lambda_{7} \\
& -4\left(5 \lambda_{1}+37 \lambda_{2}+18 \lambda_{3}+19 \lambda_{4}\right) \lambda_{5} \lambda_{7}-36 \lambda_{5}^{2} \lambda_{7}-33 \lambda_{6}^{2} \lambda_{7} \\
& -126 \lambda_{6} \lambda_{7}^{2}-111 \lambda_{7}^{3}-12\left(3 \lambda_{3}+2 \lambda_{4}+\lambda_{5}\right) \lambda_{6} y_{b}^{2} \\
& +\left\{20 g_{3}^{2}+\frac{45}{8} g_{2}^{2}+\frac{25}{24} g^{\prime 2}-6\left(3 \lambda_{3}+4 \lambda_{4}+5 \lambda_{5}\right)\right\} \lambda_{7} y_{b}^{2} \\
& +\left\{60 g_{3}^{2}+\frac{135}{8} g_{2}^{2}+\frac{85}{8} g^{\prime 2}-6\left(24 \lambda_{2}+3 \lambda_{3}+4 \lambda_{4}+5 \lambda_{5}\right)\right\} \lambda_{7} y_{t}^{2} \\
& -4\left(3 \lambda_{3}+2 \lambda_{4}+\lambda_{5}\right) \lambda_{6} y_{\tau}^{2}+\left\{\frac{5}{8}\left(3 g_{2}^{2}+5 g^{\prime 2}\right)-2\left(3 \lambda_{3}+4 \lambda_{4}+5 \lambda_{5}\right)\right\} \lambda_{7} y_{\tau}^{2} \\
& \left.-\frac{1}{4}\left(33 y_{t}^{4}+27 y_{b}^{4}+9 y_{\tau}^{4}\right) \lambda_{7}-21 \lambda_{7} y_{b}^{2} y_{t}^{2}\right] \text {. }
\end{aligned}
$$

The two-loop RGE for the soft mass parameter is given by

$$
\begin{aligned}
\mathscr{D}\left(m_{12}^{2}\right)= & \frac{1}{16 \pi^{2}}\left[-\frac{3}{2}\left(3 g_{2}^{2}+g^{2}\right) m_{12}^{2}+2\left(\lambda_{3}+2 \lambda_{4}+3 \lambda_{5}\right) m_{12}^{2}\right. \\
& \left.+2\left(3 y_{b}^{2}+3 y_{t}^{2}+y_{\tau}^{2}\right) m_{12}^{2}+12\left(\lambda_{6} \mu_{1}^{2}+\lambda_{7} \mu_{2}^{2}\right)\right] \\
& +\frac{1}{256 \pi^{4}}\left[-\frac{1}{16}\left(243 g_{2}^{4}-30 g_{2}^{2} g^{\prime 2}-153 g^{\prime 4}\right) m_{12}^{2}+3\left\{2 \lambda_{1}^{2}+\lambda_{2}^{2}\right)+\lambda_{5}^{2}\right. \\
+ & \left.4\left(\lambda_{6}^{2}+\lambda_{7}^{2}\right)\right\} m_{12}^{2}+4\left(3 g_{2}^{2}+g^{2}\right)\left(\lambda_{3}+2 \lambda_{4}+3 \lambda_{5}\right) m_{12}^{2} \\
& -12\left(\lambda_{1}+\lambda_{2}\right) \lambda_{345} m_{12}^{2}-6\left(\lambda_{3} \lambda_{4}+2 \lambda_{3} \lambda_{5}+2 \lambda_{4} \lambda_{5}+6 \lambda_{6} \lambda_{7}\right) m_{12}^{2}
\end{aligned}
$$




$$
\begin{aligned}
& +\left\{20 g_{3}^{2}+\frac{45}{8} g_{2}^{2}+\frac{25}{24} g^{\prime 2}-6\left(\lambda_{3}+2 \lambda_{4}+3 \lambda_{5}\right)\right\} y_{b}^{2} m_{12}^{2} \\
& +\left\{20 g_{3}^{2}+\frac{45}{8} g_{2}^{2}+\frac{85}{24} g^{\prime 2}-6\left(\lambda_{3}+2 \lambda_{4}+3 \lambda_{5}\right)\right\} y_{t}^{2} m_{12}^{2} \\
& +\left\{\frac{5}{8}\left(3 g_{2}^{2}+5 g^{\prime 2}\right)-2\left(\lambda_{3}+2 \lambda_{4}+3 \lambda_{5}\right)\right\} y_{\tau}^{2} m_{12}^{2} \\
& +24\left(3 g_{2}^{2}+g^{\prime 2}\right)\left(\lambda_{6} \mu_{1}^{2}+\lambda_{7} \mu_{2}^{2}\right)-72\left(\lambda_{1} \lambda_{6} \mu_{1}^{2}+\lambda_{2} \lambda_{7} \mu_{2}^{2}\right) \\
& -12 \lambda_{345}\left\{\left(2 \lambda_{6}+\lambda_{7}\right) \mu_{1}^{2}+\left(\lambda_{6}+2 \lambda_{7}\right) \mu_{2}^{2}\right\} \\
& \left.-24\left\{\left(3 y_{b}^{2}+y_{\tau}^{2}\right) \lambda_{6} \mu_{1}^{2}+3 y_{t}^{2} \lambda_{7} \mu_{2}^{2}\right\}-\frac{9}{4}\left(3 y_{b}^{4}+3 y_{t}^{4}+y_{\tau}^{4}\right) m_{12}^{2}\right]
\end{aligned}
$$

Finally, the mass parameters $\mu_{1,2}^{2}$ are removed by the tadpole conditions:

$$
\begin{aligned}
& \frac{\partial V}{\partial v_{1}}=0=-\mu_{1}^{2} v_{1}-m_{12}^{2} v_{2}+\frac{1}{2}\left[2 \lambda_{1} v_{1}^{3}+\lambda_{345} v_{1} v_{2}^{2}+3 \lambda_{6} v_{1}^{2} v_{2}+\lambda_{7} v_{2}^{3}\right] \\
& \frac{\partial V}{\partial v_{2}}=0=-\mu_{2}^{2} v_{2}-m_{12}^{2} v_{1}+\frac{1}{2}\left[2 \lambda_{2} v_{2}^{3}+\lambda_{345} v_{2} v_{1}^{2}+\lambda_{6} v_{1}^{3}+3 \lambda_{7} v_{2} v_{1}^{2}\right]
\end{aligned}
$$

and hence, it is not necessary to write down their RGEs explicitly.

\section{References}

[1] G. Aad et al. [ATLAS Collaboration], Phys. Lett. B 716, 1 (2012); S. Chatrchyan et al. [CMS Collaboration], Phys. Lett. B 716, 30 (2012).

[2] P. W. Higgs, Phys. Rev. Lett. 13, 508 (1964); F. Englert and R. Brout, Phys. Rev. Lett. 13, 321 (1964); G. S. Guralnik, C. R. Hagen and T. W. B. Kibble, Phys. Rev. Lett. 13, 585 (1964).

[3] The ATLAS and CMS Collaborations, ATLAS-CONF-2015-044.

[4] For a review, see e.g., G. C. Branco, P. M. Ferreira, L. Lavoura, M. N. Rebelo, M. Sher and J. P. Silva, Phys. Rept. 516, 1 (2012).

[5] R. A. Battye, G. D. Brawn and A. Pilaftsis, JHEP 1108, 020 (2011).

[6] ATLAS collaboration, ATLAS-CONF-2014-010; V. Khachatryan et al. [CMS Collaboration], Phys. Rev. D 90, 112013 (2014).

[7] D. Chowdhury and O. Eberhardt, arXiv:1503.08216 [hep-ph].

[8] H. Georgi and D. V. Nanopoulos, Phys. Lett. B 82, 95 (1979).

[9] J. F. Gunion and H. E. Haber, Phys. Rev. D 67, 075019 (2003).

[10] M. Carena, I. Low, N. R. Shah and C. E. M. Wagner, JHEP 1404, 015 (2014); M. Carena, H. E. Haber, I. Low, N. R. Shah and C. E. M. Wagner, Phys. Rev. D 91, 035003 (2015).

[11] P. S. B. Dev and A. Pilaftsis, JHEP 1412, 024 (2014); J. Phys. Conf. Ser. 631, 012030 (2015).

[12] D. Das and I. Saha, Phys. Rev. D 91, 095024 (2015); J. Bernon, J. F. Gunion, H. E. Haber, Y. Jiang and S. Kraml, Phys. Rev. D 92, 075004 (2015).

[13] P. H. Chankowski, T. Farris, B. Grzadkowski, J. F. Gunion, J. Kalinowski and M. Krawczyk, Phys. Lett. B 496, 195 (2000); I. F. Ginzburg and M. Krawczyk, Phys. Rev. D 72, 115013 (2005); A. Delgado, G. Nardini and M. Quiros, JHEP 1307, 054 (2013); G. Bhattacharyya and D. Das, Phys. Rev. D 91, 015005 (2015). 
[14] H. E. Haber and R. Hempfling, Phys. Rev. D 48, 4280 (1993); A. Pilaftsis and C. E. M. Wagner, Nucl. Phys. B 553, 3 (1999).

[15] M. Maniatis, A. von Manteuffel, O. Nachtmann and F. Nagel, Eur. Phys. J. C 48, 805 (2006);

C. C. Nishi, Phys. Rev. D 74, 036003 (2006) [Erratum ibid. 76, 119901 (2007)].

[16] C. C. Nishi, Phys. Rev. D 83, 095005 (2011).

[17] A. Pilaftsis, Phys. Lett. B 706, 465 (2012).

[18] M. Maniatis, A. von Manteuffel and O. Nachtmann, Eur. Phys. J. C 57, 719 (2008). I. P. Ivanov, Phys. Rev. D 77, 015017 (2008); C. C. Nishi, Phys. Rev. D 77, 055009 (2008).

[19] J. F. Gunion and H. E. Haber, Phys. Rev. D 72, 095002 (2005); M. Maniatis and O. Nachtmann, JHEP 1111, 151 (2011).

[20] N. G. Deshpande and E. Ma, Phys. Rev. D 18, 2574 (1978).

[21] R. Barbieri, L. J. Hall and V. S. Rychkov, Phys. Rev. D 74, 015007 (2006).

[22] K. A. Olive et al. (Particle Data Group), Chin. Phys. C 38, 090001 (2014).

[23] R. D. Peccei and H. R. Quinn, Phys. Rev. Lett. 38, 1440 (1977).

[24] O. Eberhardt, U. Nierste and M. Wiebusch, JHEP 1307, 118 (2013); J. Baglio, O. Eberhardt, U. Nierste and M. Wiebusch, Phys. Rev. D 90, 015008 (2014).

[25] CMS Collaboration, CMS-PAS-HIG-13-026.

[26] J. Alwall et al., JHEP 1407, 079 (2014).

[27] R. D. Ball et al., Nucl. Phys. B 867, 244 (2013).

[28] N. Craig, F. D'Eramo, P. Draper, S. Thomas and H. Zhang, JHEP 1506, 137 (2015).

[29] G. Bevilacqua, M. Czakon, C. G. Papadopoulos, R. Pittau and M. Worek, JHEP 0909, 109 (2009); M. Flechl, R. Klees, M. Kramer, M. Spira and M. Ubiali, Phys. Rev. D 91, 075015 (2015).

[30] C. G. Lester and D. J. Summers, Phys. Lett. B 463, 99 (1999).

[31] S. Kanemura, H. Yokoya and Y. J. Zheng, Nucl. Phys. B 898, 286 (2015); Y. P. Kuang and L. H. Xia, Phys. Lett. B 747, 193 (2015); N. Chen, J. Li and Y. Liu, arXiv:1509.03848 [hep-ph].

[32] V. Khachatryan et al. [CMS Collaboration], JHEP 1411, 154 (2014); G. Aad et al. [ATLAS Collaboration], JHEP 1508, 105 (2015).

[33] G. Bevilacqua and M. Worek, JHEP 1207, 111 (2012).

[34] D. Paredes [ATLAS Collaboration], PhD Thesis, Université Blaise Pascal (2013), CERN-THESIS-2013-202; J. Keaveney [CMS Collaboration], arXiv:1412.4641 [hep-ex].

[35] S. Moretti and D. A. Ross, Phys. Lett. B 712, 245 (2012); A. Djouadi, L. Maiani, A. Polosa, J. Quevillon and V. Riquer, arXiv:1502.05653 [hep-ph]; B. Bhattacherjee, A. Chakraborty and A. Choudhury, arXiv:1504.04308 [hep-ph].

[36] M. E. Machacek and M. T. Vaughn, Nucl. Phys. B 222, 83 (1983); 236, 221 (1984); 249, 70 (1985).

[37] F. Staub, Comput. Phys. Commun. 185, 1773 (2014).

[38] G. Lee and C. E. M. Wagner, arXiv:1508.00576 [hep-ph]. 